\title{
A rotation-free shell triangle for the analysis of kinked and branching shells
}

\author{
Fernando G. Flores ${ }^{1, *, \dagger}$ and Eugenio Oñate ${ }^{2, \ddagger}$ \\ ${ }^{1}$ National University of Córdoba, Casilla de Correo 916, 5000 Córdoba, Argentina \\ ${ }^{2}$ International Center for Numerical Methods in Engineering, Edificio C1, Gran Capitán s/n, \\ 08034 Barcelona, Spain
}

\begin{abstract}
SUMMARY
This paper extends the capabilities of previous BST and EBST rotation-free thin shell elements to the analysis of kinked and branching surfaces. The computation of the curvature tensor is first redefined in terms of the angle change between the normals at the adjacent elements. This allows to deal with arbitrary large angles between adjacent elements and to treat kinked surfaces. A relative stiffness between elements is introduced to consider non-homogeneous surfaces. This idea is latter generalized to deal with branching shells. Several linear and non-linear examples are presented showing that the formulation leads to the correct results. Copyright (c) 2006 John Wiley \& Sons, Ltd.
\end{abstract}

Received 2 September 2005; Revised 24 April 2006; Accepted 26 May 2006

KEY WORDS: finite elements; shells; rotation-free triangle; branching shells; kinked shells; large strains

\section{INTRODUCTION}

The development of numerical techniques for thin (Kirchhoff-Love) shell analysis including only translational degrees of freedom (the so called rotation-free elements) has been mainly associated to the finite-difference method (see for example References [1-3]). Nevertheless the idea of developing finite elements for beams and shells without rotational degrees of freedom is not new and many attempts have been reported [4-11]. Despite these attempts it is just in the last few

\footnotetext{
*Correspondence to: Fernando G. Flores, National University of Córdoba, Casilla de Correo 916, 5000 Córdoba, Argentina.

†E-mail: fflores@efn.uncor.edu

${ }^{\ddagger}$ E-mail: onate@cimne.upc.edu

Contract/grant sponsor: CONICET

Contract/grant sponsor: CIMNE
}

Copyright (C) 2006 John Wiley \& Sons, Ltd. 
years that reliable rotation-free shell elements for industrial applications have been developed (see References [12-17] for a state of the art).

There are also finite elements based on transverse shear deformable shell theories that do not include rotational degrees of freedom. But the approximation used is completely different as the rotations are eliminated at the expense of new degrees of freedom, namely the displacements of the top and bottom surfaces (or the difference between them). On one hand, this maintains the number of DOFs and on the other it requires similar techniques to avoid locking phenomena as those used for the elements including rotational degrees of freedom. This type of finite elements are based on the so called 'degenerated solid approach' (a detailed discussion of this approach can be found in Reference [18]); they avoid rotations to facilitate the connection with solid elements and they typically make use of a general three-dimensional (3D) elasticity theory (thereby skipping the usual plane-stress assumptions of shell theory) which allows then to implement 3D constitutive relations [19].

The finite element models using rotation-free thin shell elements present several advantages and some limitations depending on the type of application. The main advantages are:

- For a required precision, the number of total DOFs in the model, compared with a widely used element as the DKT [20] may be less than a half. This means important savings in both storage and CPU time.

- Rotation vectors or local triads, that are in general costly and difficult to parameterize and

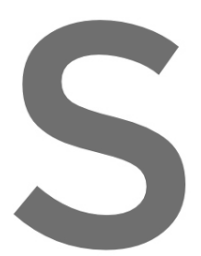

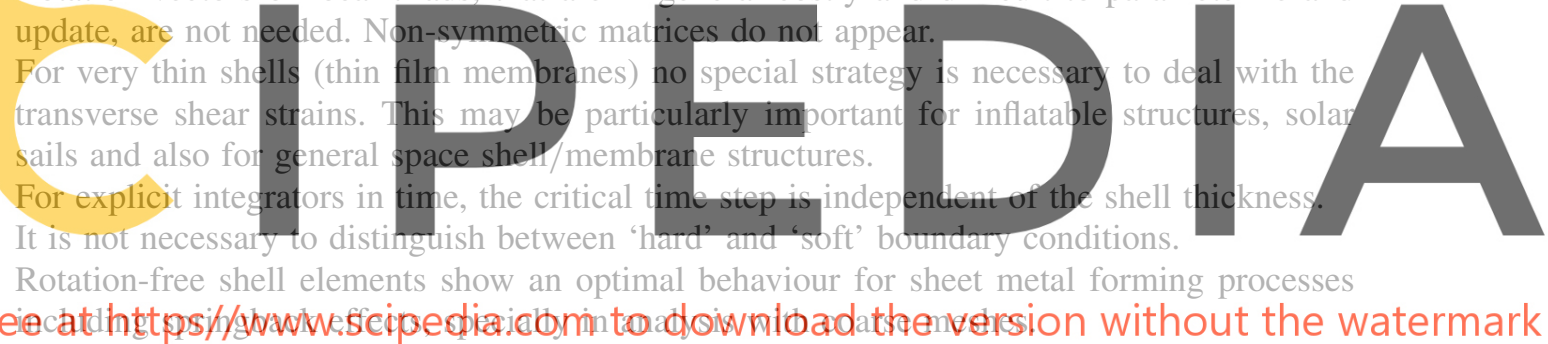

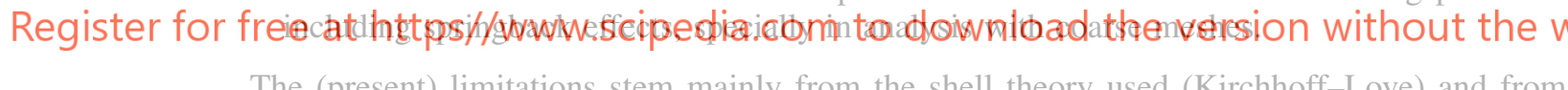

The (present) limitations stem mainly from the shell theory used (Kirchhoff-Love) and from the absence of rotational DOFs. We can mention:

- A direct combination with other finite elements, like beam or solid elements, is not straightforward. Special purpose multipoint constraints are necessary.

- Coding may be more involved.

- Transverse shear strains are disregarded. This may be an important limitation for the analysis of composite laminates.

- Plane-stress assumption precludes the use of general 3D constitutive models.

All the approximations made for rotation-free classical shell elements share in common the definition of a patch (neighbourhood) of elements to interpolate the geometry and compute the curvatures. The main difference between the different approaches is how the geometry is interpolated and the theoretical basis used to compute the membrane and bending strains. One of the main aspects that remains unsolved satisfactorily is the treatment of non-smooth surfaces and specially branching shells. An adequate handling of multiple surfaces, i.e. when more than two elements share a side or edge, is mandatory if the element is to be used for the analysis of aeronautic, civil or naval structures among others. Branching surfaces, i.e. when more than two surfaces share a side, represents the main challenge. 
In this paper 3D shell problems are tackled with special focus on non-smooth and branching shells. This work is an extension to branching shells of previous developments $[13,15]$ of rotationfree 3D shell elements. And is also an extension to 3D shells of a 2D rotation-free shell element adequate to deal with kinked and branching surfaces [21].

An outline of this paper is as follows. In Section 2, a brief summary of the main parameters defining the shell deformation is presented. In the following section the computation of the curvature for the BST element [13] is revisited and it is shown how to modify it to allow the treatment of large angle changes and non-smooth surfaces. These developments are extended to branching shells in Section 4. Section 5 considers the same developments but for the EBST element [15] using an explicitly defined quadratic interpolation of the geometry. In Section 6 the formulation is completed including the membrane part and the main aspects associated to the stiffness matrix evaluation. In the last part (Section 7) the numerical assessment of the proposed element is performed via the solution of linear, non-linear elastic and large-strain elastic-plastic problems. Some conclusions are gathered at the end of the paper.

\section{KINEMATICS OF A THIN SHELL}

In this section a brief summary of the principal hypothesis regarding the kinematic behaviour of thin shells are presented. More detaiks can be found in the wide literature dedicated to this

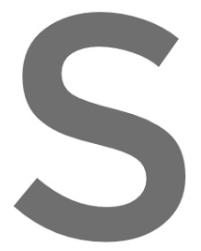

field [1].

Let us consider a Euclidean space $R^{3}$ defined as the distance the shell. The thickndss may tot ore detaits can be
whose undeformed
dary ${ }^{\circ}$. At each
ed along the norm
ot be constant but found in the w
point of this $m$
al $o_{3}$ ) between the
should have a ide literature dedicate
occupies the domain
idcle surfade a thickn
the upper and lower su
smooth valiation. The $\Omega$ in th

thin shell Kirchhoff-Love hypothesis that fibres originally orthogonal to the middle surface remain

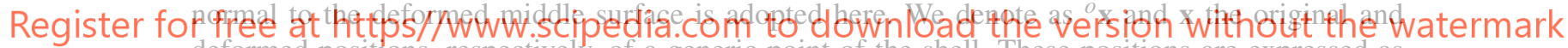
deformed positions, respectively, of a generic point of the shell. These positions are expressed as a function of the co-ordinates of the associated point on the middle surface $\varphi$ and its normal at that point $\mathbf{t}_{3}$ as

$$
\begin{gathered}
{ }^{o} \mathbf{x}\left(\xi_{1}, \xi_{2}, \xi_{3}\right)={ }^{o} \boldsymbol{\varphi}\left(\xi_{1}, \xi_{2}\right)+\xi_{3}{ }^{o} \mathbf{t}_{3}\left(\xi_{1}, \xi_{2}\right) \\
\mathbf{x}\left(\xi_{1}, \xi_{2}, \xi_{3}\right)=\boldsymbol{\varphi}\left(\xi_{1}, \xi_{2}\right)+\xi_{3} \lambda \mathbf{t}_{3}\left(\xi_{1}, \xi_{2}\right)
\end{gathered}
$$

where $\xi_{1}, \xi_{2}$ are local curvilinear co-ordinates defined over the undeformed middle surface and $\xi_{3}$ is the (original) distance of the generic point to the middle surface $\left(\xi_{3} \in\left[-{ }^{o} t / 2,{ }^{o} t / 2\right]\right)$. We will assume that the local in-plane co-ordinates $\left(\xi_{1}, \xi_{2}\right)$ define orthogonal lines (not necessarily lines of curvature) and that the associated Lamé parameters are the unity, i.e.:

$$
{ }^{o} \boldsymbol{\varphi}^{\prime} i \cdot{ }^{o} \boldsymbol{\varphi}_{\prime j}=\delta_{i j}
$$

with $\delta_{i j}$ the Kronecker delta. The motivation for such special system is directly associated to the numerical implementation where a local Cartesian system with the same properties is defined at each integration point. The product $\xi_{3} \lambda$ is the distance from the point to the middle surface in the deformed configuration. This implies a constant transversal stretch associated to the parameter $\lambda$ 
relating the thickness at the deformed configuration and the original thickness, i.e.

$$
\lambda=\frac{t}{o_{t}}
$$

The deformation gradient, defined as the derivative of (2) with respect to the local co-ordinates $\xi_{i}$, can be expressed as

$$
\mathbf{F}=\left[\boldsymbol{\varphi}_{11}+\xi_{3}\left(\lambda \mathbf{t}_{3}\right)_{\prime 1} \boldsymbol{\varphi}_{2}+\xi_{3}\left(\lambda \mathbf{t}_{3}\right)^{\prime 2} \lambda \mathbf{t}_{3}\right]
$$

The product $\mathbf{F}^{\mathrm{T}} \mathbf{F}=\mathbf{U}^{2}=\mathbf{C}$ (where $\mathbf{U}$ is the right stretch tensor and $\mathbf{C}$ the right Cauchy-Green tensor) results in

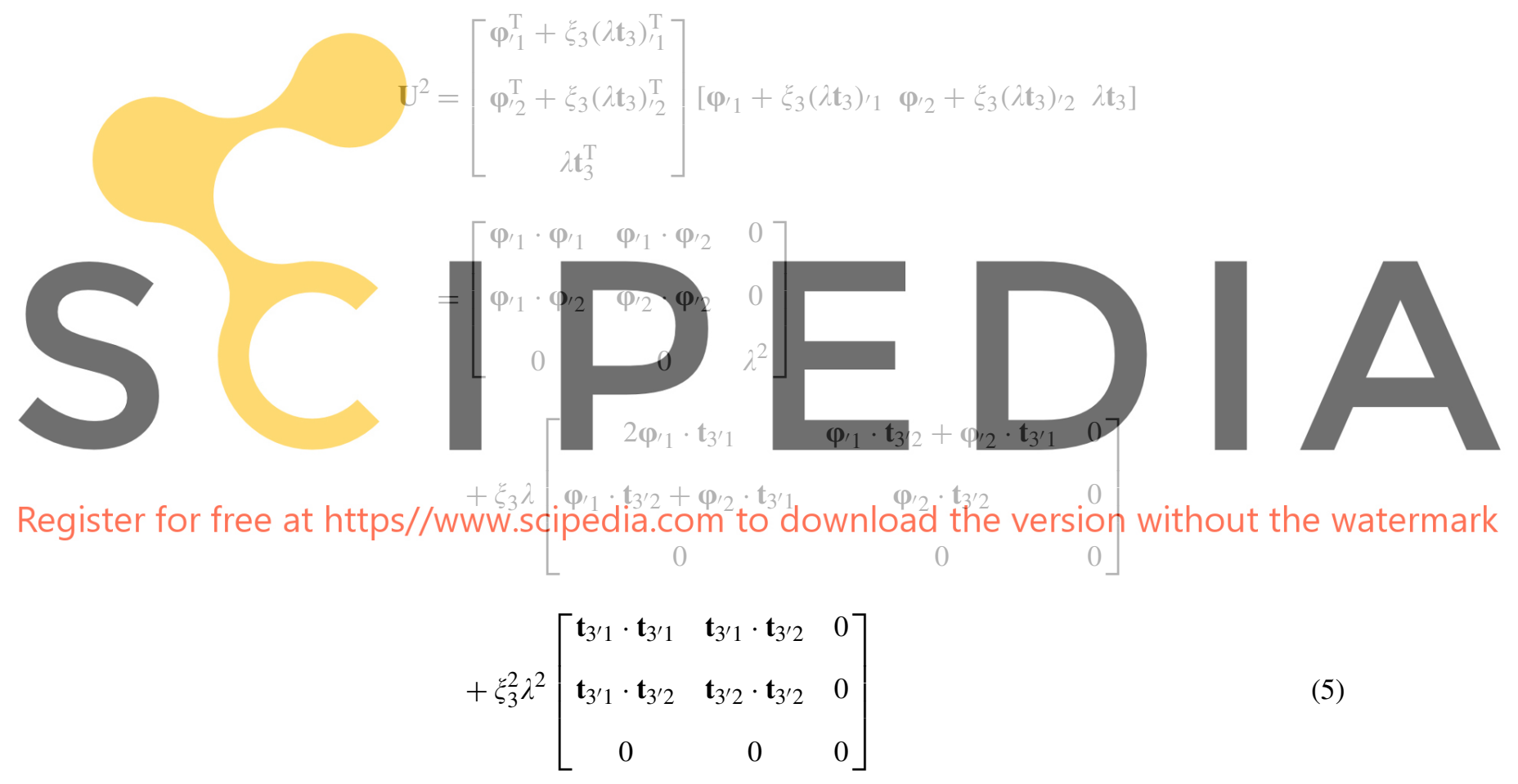

where the influence of the derivatives of the thickness ratio $\lambda_{a}$ have been neglected. Disregarding also the terms associated with $\xi_{3}^{2}$ and introducing the following definitions of the first and second fundamental forms of the surface (with $\alpha, \beta=1,2$ ):

(a) the covariant metric tensor or first fundamental form of the middle surface:

$$
a_{\alpha \beta}=\boldsymbol{\varphi}_{\prime \alpha} \cdot \boldsymbol{\varphi}^{\prime} \beta
$$

(b) the curvature tensor or second fundamental form of the middle surface:

$$
\kappa_{\alpha \beta}=\frac{1}{2}\left(\boldsymbol{\varphi}^{\prime} \alpha \cdot \mathbf{t}_{3^{\prime} \beta}+\boldsymbol{\varphi}_{\beta} \beta \cdot \mathbf{t}_{3^{\prime} \alpha}\right)
$$


the right stretch tensor can be written as

$$
\mathbf{U}^{2}=\left[\begin{array}{ccc}
a_{11}+2 \kappa_{11} \xi_{3} \lambda & a_{12}+2 \kappa_{12} \xi_{3} \lambda & 0 \\
a_{12}+2 \kappa_{12} \xi_{3} \lambda & a_{22}+2 \kappa_{22} \xi_{3} \lambda & 0 \\
0 & 0 & \lambda^{2}
\end{array}\right]
$$

Note that for the special local co-ordinate system used, the metric tensor at the original configuration ${ }^{o} a_{\alpha \beta}$ is the unit tensor. But for initially curved surfaces ${ }^{o} \mathbf{F}$ and ${ }^{o} \mathbf{U}^{2}$ are not unit tensors for points outside the middle surface, also the Jacobian (determinant of ${ }^{o} \mathbf{F}$ ) is not constant across the thickness.

Introducing the change of curvature tensor

$$
\chi_{\alpha \beta}=\kappa_{\alpha \beta}-{ }^{o} \kappa_{\alpha \beta}
$$

The right Cauchy-Green tensor in Equation (8) can be approximated by the following expression, that is computationally more convenient because it is the unit tensor everywhere at the original configuration:

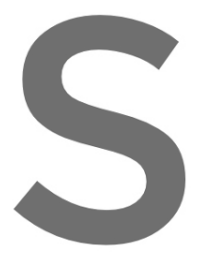

A

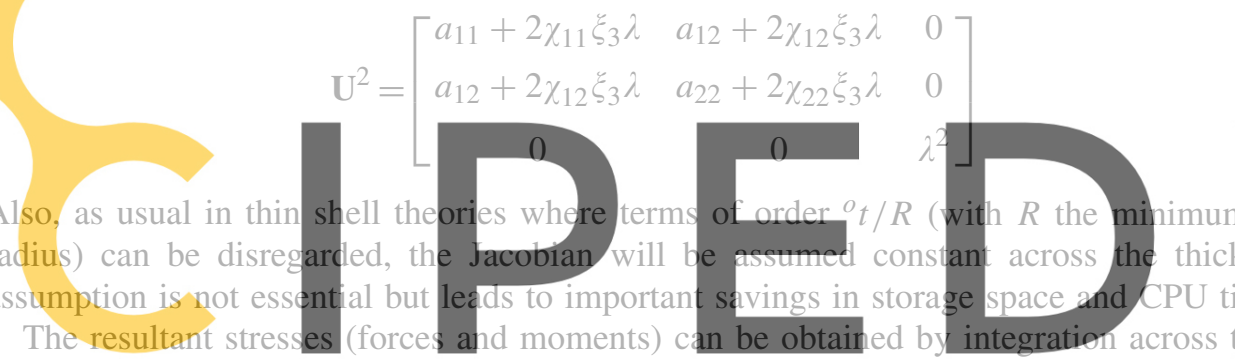

thickness of the second Piola-Kirchhoff stress tensor S:

$$
\begin{aligned}
& \text { Register for free at https//www.scipedia.com tion download the version without the watermark } \\
& \mathbf{M}=\int_{-{ }^{o} t / 2}^{o t / 2} \mathbf{S} \lambda \xi_{3} \mathrm{~d} \xi_{3}
\end{aligned}
$$

(10)

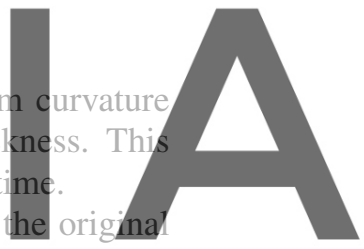

where $\lambda \xi_{3}$ is the actual distance of the point to the shell middle surface and a constant Jacobian is assumed across the thickness.

The weak form of the equilibrium equations can be written as

$$
\delta \Pi=\int_{o_{\Omega}}[\delta \mathbf{E}: \mathbf{N}+\delta \mathbf{K}: \mathbf{M}] \mathrm{d}^{o} \Omega+\delta \Pi_{\mathrm{ext}}=0
$$

where $\delta \mathbf{K}$ is the variation of the curvature tensor and $\delta \mathbf{E}$ is the variation of the Green-Lagrange strain tensor at the middle surface

$$
\begin{aligned}
\mathbf{E} & =\frac{1}{2}\left(\mathbf{U}^{2}-\mathbf{1}\right) \\
E_{\alpha \beta} & =\frac{1}{2}\left(a_{\alpha \beta}-\delta_{\alpha \beta}\right)
\end{aligned}
$$

and $\delta_{\alpha \beta}$ is the Kronecker delta. 


\section{BST ELEMENT FOR NON-SMOOTH SURFACES}

One of the main features of the rotation-free shell finite elements is that they are non-conforming as the continuity of the normal $\mathbf{t}_{3}$ is not assured across the element boundary. To restore the continuity of the rotations between elements at discrete points and for the evaluation of the curvatures a patch of elements is used. This patch (shown in Figure 1(a)) includes the element under consideration and the adjacent ones. The figure shows the local numbering for the nodes and the sides. The same patch is shown in Figure 1(b) over a normalized domain (master element). Note the numbers assigned to nodes and sides:

- nodes in the main element are numbered from 1 to 3 ; node 4 is opposite to node 1 , node 5 is opposite to node 2 and node 6 to node 3 ;

- side $i$ is the side opposite to node $i$ in the main element; and the element $i$ is the element adjacent to that side;

- the connectivity at each adjacent element $i$ begin with the extra node $(i+3)$.

\subsection{Computation of curvatures for element BST}

The computation of the curvatures (7) for the BST element [13], where it is assumed that the four elements in the patch belong to a smooth surface, stems from the average integral (15a) and its
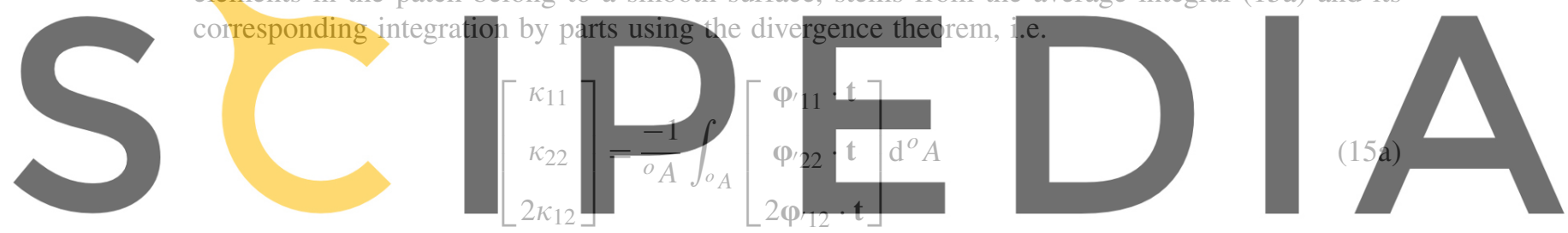

Register for free at https//www.scipedia.com $\prod^{\text {to }} \mathrm{d} d \boldsymbol{b}$ winload the version without the watermark $=\frac{-1}{o_{A}} \int_{o \Gamma}\left[\begin{array}{cc}0 & n_{2} \\ n_{2} & n_{1}\end{array}\right]\left\lfloor\begin{array}{c}\varphi^{\prime} 1 \\ \boldsymbol{\varphi}^{\prime} 2 \\ \mathbf{o t}^{(M)}\end{array}\right] \mathrm{d}^{O} \Gamma$

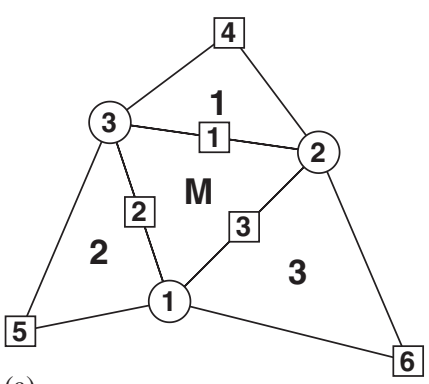

(a)

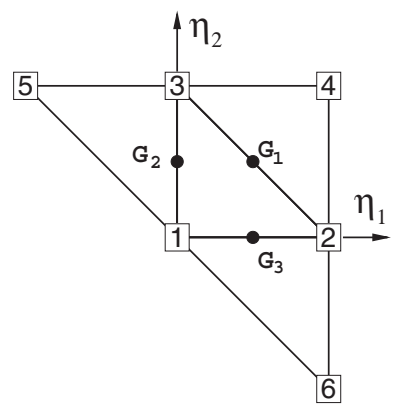

(b)

Figure 1. Patch of three-noded triangular elements including the main triangle (M) and the three adjacent ones (1, 2 y 3$)$. 
where ${ }^{o} A$ is the original area of the main element and ${ }^{o} \Gamma$ its boundary with normal ${ }^{o} \mathbf{n}=\left({ }^{o} n_{1},{ }^{o} n_{2}\right)^{\mathrm{T}}$. The directions 1 and 2 used to express the components of the curvature tensor $\kappa_{i j}$ and the outward normal ${ }^{o} n_{i}$ define an arbitrary local orthogonal Cartesian system over the original surface. Unit vector $\mathbf{t}^{(M)}$ is the (present) normal to the element (defined by its three nodes).

With an upper-index between round brackets we will denote, when necessary, the element in the patch of the associated geometrical parameter. For example $\mathbf{t}^{(M)}$ is the normal to the main element, and $\mathbf{t}^{(i)}$ is the normal to the adjacent element opposite to node $i$ of the main triangle. The geometry of the middle surface $\varphi$ is interpolated independently at each element from its three nodes $\varphi^{I}$ using standard linear functions $L^{I}$ in terms of area co-ordinates $\left(\eta_{1}, \eta_{2}\right)$ [22] (see Figure 1):

$$
\varphi=\sum_{I=1}^{3} L^{I}\left(\eta_{1}, \eta_{2}\right) \varphi^{I}
$$

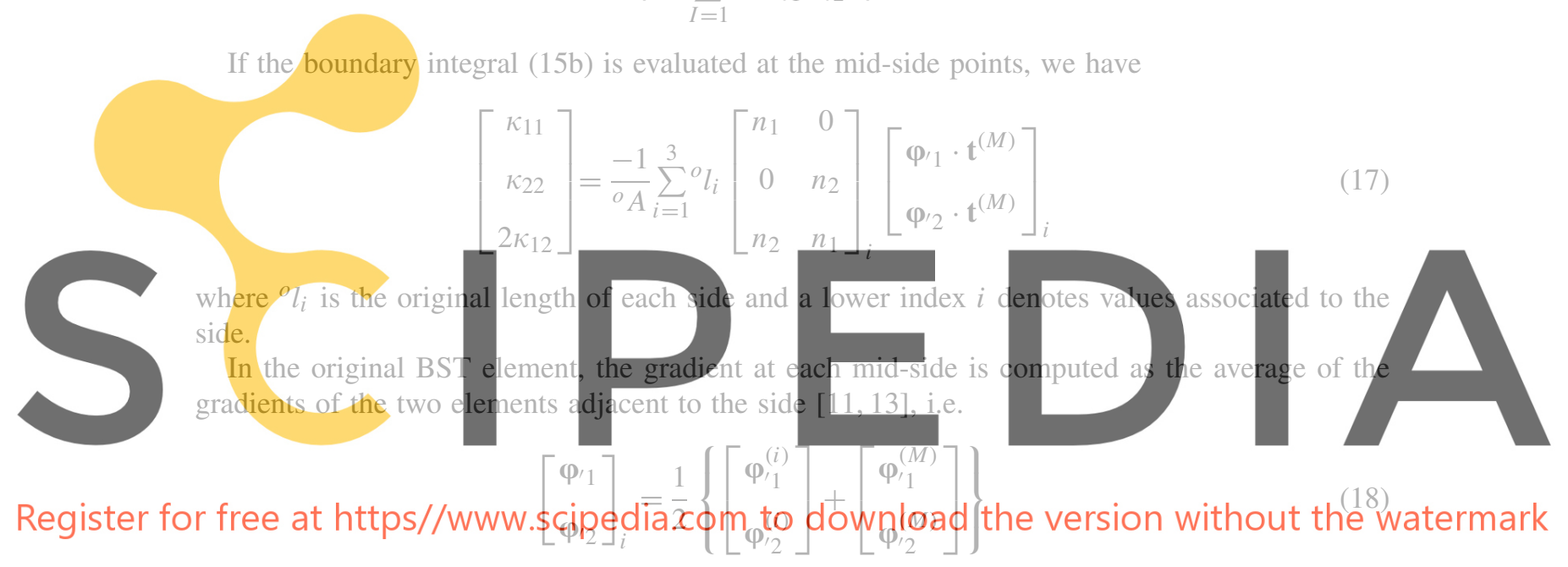

It must be also noted that

- on the main element $\left[\begin{array}{l}\boldsymbol{\varphi}_{1}^{(M)} \cdot \mathbf{t}^{(M)} \\ \boldsymbol{\varphi}_{/ 2}^{(M)} \cdot \mathbf{t}^{(M)}\end{array}\right]=\left[\begin{array}{l}0 \\ 0\end{array}\right]$

- resolving the gradient on the adjacent element over a local system at the common side $\left({ }^{o} \mathbf{n},{ }^{o} \mathbf{s}\right)$ : $\left[\boldsymbol{\varphi}_{1}^{(i)}, \boldsymbol{\varphi}_{2}^{(i)}\right]=\left[\boldsymbol{\varphi}_{n}^{(i)}, \boldsymbol{\varphi}_{{ }_{s}}^{(i)}\right]\left[\begin{array}{cc}{ }^{o}{ }^{o} n_{1} & -{ }^{o} n_{2} \\ { }^{o} n_{2} & -{ }^{o} n_{1}\end{array}\right]_{i}$ we have $\boldsymbol{\varphi}_{s_{s}}^{(i)} \cdot \mathbf{t}^{(M)}=0$

Then

$$
\left[\begin{array}{c}
\kappa_{11} \\
\kappa_{22} \\
2 \kappa_{12}
\end{array}\right]=\frac{1}{2^{o} A} \sum_{i=1}^{3}{ }^{o} l_{i}\left[\begin{array}{cc}
{ }^{o} n_{1} & 0 \\
0 & { }^{o} n_{2} \\
{ }^{o} n_{2} & { }^{o} n_{1}
\end{array}\right]_{i}\left[\begin{array}{c}
{ }^{o} n_{1} \\
{ }^{o} n_{2}
\end{array}\right]_{i}\left(\boldsymbol{\varphi}_{{ }_{n}}^{(i)} \cdot \mathbf{t}^{(M)}\right)
$$

Introducing the derivatives of the linear triangle shape functions $L^{i}$ [22] as

$$
\left[\begin{array}{c}
L_{\prime 1}^{i} \\
L_{\prime 2}^{i}
\end{array}\right]=-\frac{{ }^{o} l_{i}}{2^{o} A}\left[\begin{array}{c}
o \\
n_{1} \\
{ }^{o} n_{2}
\end{array}\right]_{i}=-\frac{1}{{ }^{o} h_{i}}\left[\begin{array}{c}
{ }^{o} n_{1} \\
{ }^{o} n_{2}
\end{array}\right]_{i}
$$


where ${ }^{o} h_{i}$ is the distance from node $i$ to the its opposite side. Equation (19) can be rewrited as

$$
\left[\begin{array}{c}
\kappa_{11} \\
\kappa_{22} \\
2 \kappa_{12}
\end{array}\right]=2^{o} A \sum_{i=1}^{3} \frac{1}{o} l_{i}\left[\begin{array}{c}
\left(L_{\prime_{1}}^{i}\right)^{2} \\
\left(L_{\prime_{2}}^{i}\right)^{2} \\
-2 L_{\prime_{1}}^{i} L_{\prime_{2}}^{i}
\end{array}\right]\left(\boldsymbol{\varphi}_{{ }_{n}}^{(i)} \cdot \mathbf{t}^{(M)}\right)
$$

Expression (21) or the equivalent one in terms of the gradient components (17) are used in the BST element to compute the curvatures in the analysis of smooth surfaces. Note that the projection $\varphi_{/ n}^{(i)} \cdot \mathbf{t}^{(M)}$ is approximately equal to the sine of the angle between the normals. In fact this approximation is valid for small angles between element normals but for arbitrary large angles as normally occurs in kinked and branching surfaces the angle itself must be used [16,21] instead of the sine of the angle in the curvature definition (21).

With this target in mind, if $2 \gamma_{i}$ is the angle between the normal at the main element and the normal at the adjacent element $i$, the projection $\varphi_{! n}^{(i)} \cdot \mathbf{t}^{(M)}$ can be alternatively interpreted as (see Appendix A)
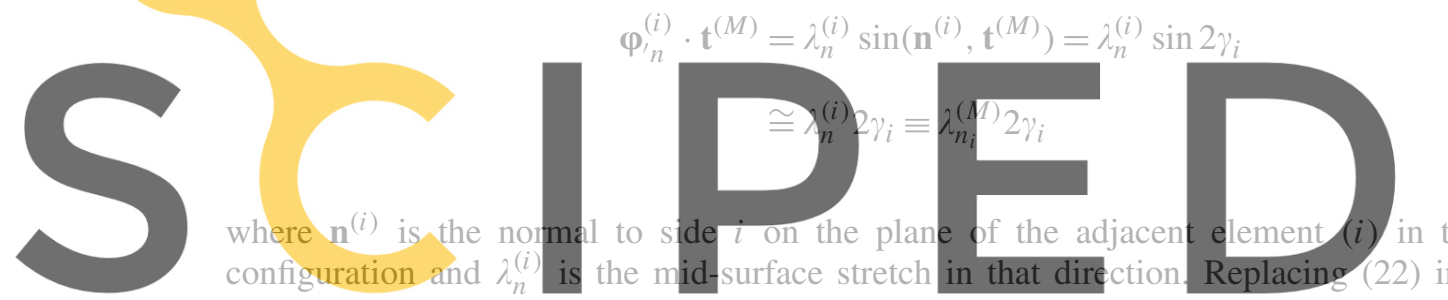

expression (computationally more expensive than 21) for computing the element curvatures is

expression (computationally more expensive than 21) for computing the element curvatures is

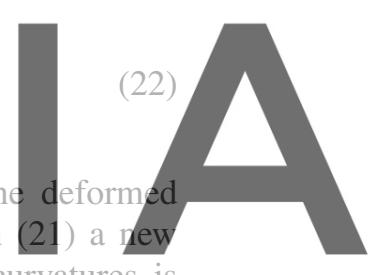

Register for free at https//www.scipedia.com to download the version without the watermark

$$
\left[\begin{array}{c}
\kappa_{11} \\
\kappa_{22} \\
2 \kappa_{12}
\end{array}\right]=2^{o} A \sum_{i=1}^{3} \frac{1}{o} l_{i}\left[\begin{array}{c}
\left(L_{\prime_{1}}^{i}\right)^{2} \\
\left(L_{\prime_{2}}^{i}\right)^{2} \\
-2 L_{\prime_{1}}^{i} L_{\prime_{2}}^{i}
\end{array}\right] \lambda_{n_{i}}^{(M)} 2 \gamma_{i}
$$

This redefinition of the element curvatures will be used below to deal with non-smooth shells. The variation of the curvature tensor components, necessary for the computation of weak form of the momentum equations (12), is

$$
\delta\left[\begin{array}{c}
\kappa_{11} \\
\kappa_{22} \\
2 \kappa_{12}
\end{array}\right]=2^{o} A \sum_{i=1}^{3} \frac{1}{o} l_{i}\left[\begin{array}{c}
\left(L_{\prime_{1}}^{i}\right)^{2} \\
\left(L_{\prime_{2}}^{i}\right)^{2} \\
-2 L_{\prime_{1}}^{i} L_{\prime_{2}}^{i}
\end{array}\right] \delta\left(\lambda_{n_{i}}^{(M)} 2 \gamma_{i}\right)
$$

In the next two sections we will see how to compute (23) and (24) for shells with kinks and branches. It is of particular interest the approximation and computation of $\lambda_{n_{i}}^{(M)} 2 \gamma_{i}$ and its variation over each side $i$ of the main element of the patch. 


\subsection{Non-smooth surfaces}

Based on previous developments of a rotation-free one-dimensional shell element [21], this section is devoted to extend the BST element capabilities to shells with discontinuities in the normal direction (non-smooth surfaces).

Let us suppose that in the original configuration a non-zero angle ${ }^{o} \phi_{i}$ exists between the normals of two adjacent elements. Over each element, on the common side, it is possible to define a local triad with: the element normal ${ }^{o} \mathbf{t}$, a unit vector along the common side ${ }^{o} \mathbf{s}$ and the normal to the side in the element plane ${ }^{o} \mathbf{n}={ }^{o} \mathbf{s} \times{ }^{o} \mathbf{t}$, so that

$$
\begin{aligned}
& \cos { }^{o} \phi_{i}={ }^{o} \mathbf{t}^{(M)} \cdot{ }^{o} \mathbf{t}^{(i)}=-{ }^{o} \mathbf{n}_{i}^{(M)} \cdot{ }^{o} \mathbf{n}^{(i)} \\
& \sin ^{o} \phi_{i}={ }^{o} \mathfrak{t}^{(M)} \cdot{ }^{o} \mathrm{n}^{(i)}={ }^{o} \mathrm{n}_{i}^{(M)} \cdot{ }^{o} \mathfrak{t}^{(i)}
\end{aligned}
$$

Recall that the upper index indicates the element and the lower index the side. However, for the parameters computed at the element $i$ the lower index $i$ is suppressed to alleviate the notation. For example ${ }^{o} \mathbf{n}^{(i)}$ is the normal to side $i$ over the tangent plane at element $i$. Note that: (a) ${ }^{o} \mathbf{s}_{i}^{(M)} \equiv{ }^{o} \mathbf{S}_{i}$ and ${ }^{o} \mathbf{S}^{(i)}$ are along the same line but have opposite directions; (b) with the previous notation the angle ${ }^{o} \phi_{i}$ is measured around the common side ${ }^{o} \mathbf{s}_{i}$ counter-clockwise from ${ }^{o} \mathbf{t}^{(M)}$ to ${ }^{o} \mathbf{t}^{(i)}$; (c) the initial angle ${ }^{o} \phi_{i}$ (25) between the normal vectors to the two elements is also the angle between

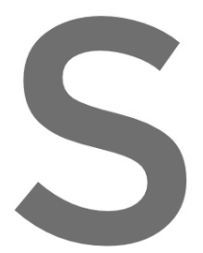
the normal vectors to the common side oyer the tangent planes and $\boldsymbol{-}^{\circ} \mathbf{n}^{(i)}$ ) (Figure 2).

Using the linear mappi (i.e. the tangent planes computed for each element:
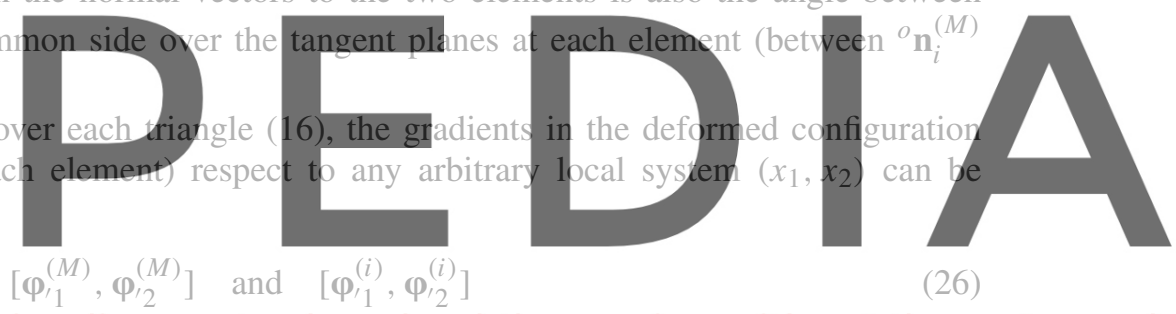

Register for frefe at https. (Lwww scipedia.com to download the version without the watermark

$$
\mathfrak{t}^{(M)}=\lambda^{(M)} \varphi_{1}^{(M)} \times \varphi_{2}^{(M)}, \quad \mathfrak{t}^{(i)}=\lambda^{(i)} \varphi_{1}^{(i)} \times \varphi_{2}^{(i)}
$$

The common side written in terms of the present unit vector along the side $\mathbf{s}_{i}$ and the stretch of the fibre $\lambda_{s}$

$$
\boldsymbol{\varphi}_{{ }^{\prime}}=\lambda_{s} \mathbf{s}_{i}=\boldsymbol{\varphi}_{{ }_{s}}^{(M)}=-\boldsymbol{\varphi}_{{ }_{s}}^{(i)}
$$

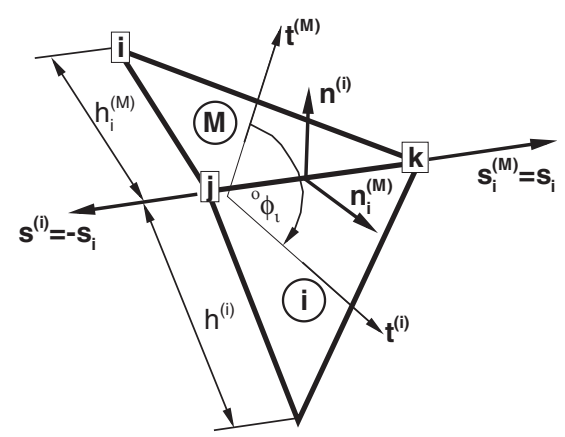

Figure 2. Angle between two elements. 
allows to compute the normal vectors to the side over each element plane

$$
\begin{aligned}
\mathbf{n}_{i}^{(M)} & =\frac{1}{\lambda_{s}} \boldsymbol{\varphi}_{s_{s}}^{(M)} \times \mathbf{t}^{(M)}=\mathbf{s}_{i} \times \mathbf{t}^{(M)} \\
\mathbf{n}^{(i)} & =\frac{1}{\lambda_{s}} \boldsymbol{\varphi}_{s_{s}}^{(i)} \times \mathbf{t}^{(i)}=-\mathbf{s}_{i} \times \mathbf{t}^{(i)}
\end{aligned}
$$

Note also that in the original configuration the in-plane normal to the side is equivalent to the gradient in that direction, but not in the deformed configuration.

The normal vectors $\mathbf{t}^{(M)}$ and $\mathbf{t}^{(i)}$ (and also $\mathbf{n}_{i}^{(M)}$ and $\mathbf{n}^{(i)}$ ) in the deformed configuration will now form an angle $\phi_{i}$

$$
\begin{aligned}
& \cos \phi_{i}=\mathbf{t}^{(M)} \cdot \mathbf{t}^{(i)}=-\mathbf{n}_{i}^{(M)} \cdot \mathbf{n}^{(i)} \\
& \sin \phi_{i}=\mathbf{t}^{(M)} \cdot \mathbf{n}^{(i)}=\mathbf{n}_{i}^{(M)} \cdot \mathbf{t}^{(i)}
\end{aligned}
$$

this represents a change of angle respect to the original configuration:
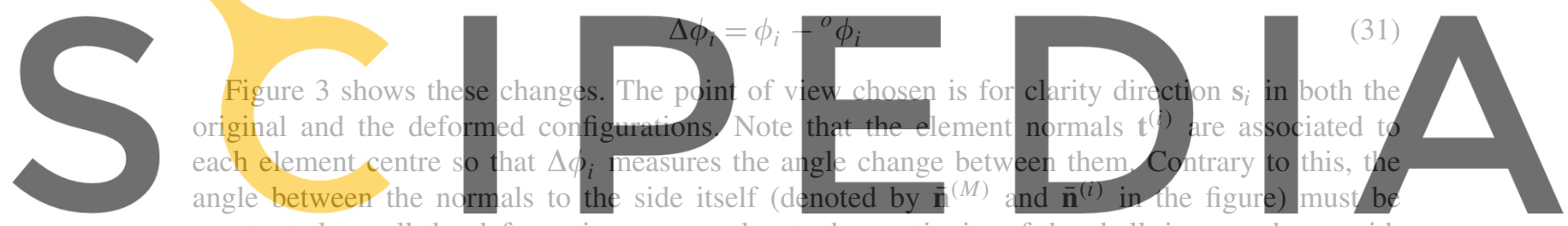

constant along all the deformation process due to the continuity of the shell, in accordance with

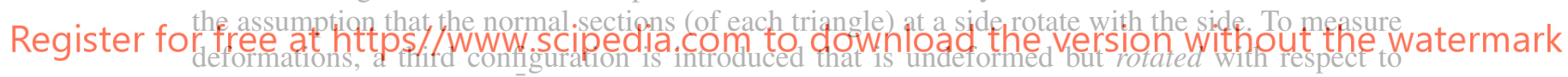

the original one an angle $\bar{\beta}_{i}$ which is defined as the rotation of the side $\mathbf{s}_{i}$ around itself.

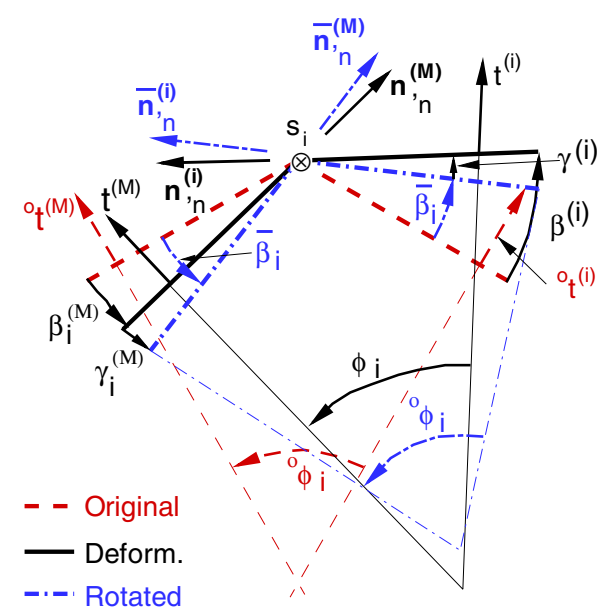

Figure 3. Configurations used for the definition of the relative rotations $\gamma^{(i)}$. 
To maintain the original angle ${ }^{o} \phi_{i}$ between the normals to the side $\overline{\mathbf{n}}^{(M)}$ and $\overline{\mathbf{n}}^{(i)}$ it is possible to associate to each element a relative rotation $\gamma^{()}$of its normal $\mathbf{t}^{()}$respect to the rotation of the side $\bar{\beta}_{i}$, with the condition that

$$
\Delta \phi_{i}=\gamma_{i}^{(M)}+\gamma^{(i)}
$$

For a smooth homogeneous shells of uniform thickness $[13,15,16]$ the angles $\gamma_{i}^{(M)}$ and $\gamma^{(i)}$ are assigned values proportional to the distance from the side to the opposite node in each element.

$$
\frac{\gamma_{i}^{(M)}}{h_{i}^{(M)}}=\frac{\gamma^{(i)}}{h^{(i)}}
$$

in the case of $2 \mathrm{D}$ beams this can be shown to be equivalent to equate the normal curvature on both sides or use bending equilibrium [21]. Following this idea, to obtain the values of $\gamma^{()}$Equation (32) will be supplemented with an approximate elastic bending equilibrium equation around the side (an approximate equilibrium as it does not include the curvature along the side and the relation $\gamma / h$ is not strictly the normal curvature)

$$
\begin{aligned}
\left(\frac{E t^{3}}{\left(1-v^{2}\right)} \frac{1}{h}\right)^{(M)} \gamma_{i}^{(M)} & =\left(\frac{E t^{3}}{\left(1-v^{2}\right)} \frac{1}{h}\right)^{(i)} \gamma^{(i)} \\
R_{i}^{(M)} \gamma_{i}^{(M)} & =R^{(i)} \gamma^{(i)}
\end{aligned}
$$

where $E$ is the modulus of elasticity, $v$ is the Poisson ratio, $t$ the shell thickness and $h$ the height of the triangle (measured from the common side) of each adjacent element. That leads to

$$
\begin{gathered}
\gamma_{i}^{(M)}=\frac{R^{(i)}}{R_{i}^{(M)}+R^{(i)}} \Delta \phi_{i}=r_{i}^{(M)} \Delta \phi_{i} \\
\gamma^{(i)}=\frac{R^{(M)}}{R_{i}^{(M)}+R^{(i)}} \Delta \phi_{i}=r^{(i)} \Delta \phi_{i}
\end{gathered}
$$

Equation (34) is the main assumption introduced to extend the rotation-free shell elements to non-smooth non-homogeneous shells, and this assumption will be later extended to multiple surface intersection. The approximation may look rather strong but the numerical results obtained are remarkably good.

Assuming for an instant side direction $\mathbf{s}_{i}$ fixed in the space and denoting by:

- $\beta_{i}^{(M)}$ the angle rotated by element $(M)$ expressed as the angle between $\mathbf{t}^{(M)}$ and ${ }^{o} \mathbf{t}^{(M)}$ (measured from ${ }^{o} \mathbf{t}^{(M)}$ to ${ }^{o} \mathbf{t}^{(M)}$ counter-clockwise around $\mathbf{s}_{i}$ )

- $\beta^{(i)}$ the angle rotated by the adjacent element $i$, expressed as the angle between $\mathbf{t}^{(i)}$ and ${ }^{o} \mathbf{t}^{(i)}$ (measured from ${ }^{o} \mathbf{t}^{(i)}$ to $\mathbf{t}^{(i)}$ clockwise around $\mathbf{s}^{(i)}$, equivalent to measure it counter-clockwise around $\mathbf{s}_{i}$ ) 
(note that both angles are measured around the same axis) from expressions (36) the angle $\Delta \phi_{i}$ can be also seen as the difference between the angles rotated by each element around the side (using direction $\mathbf{s}_{i}$ as reference), i.e.

$$
\Delta \phi_{i}=\beta^{(i)}-\beta_{i}^{(M)}
$$

Finally it is possible to define the angle rotated by the side as the weighted average of the angles rotated by each element

$$
\bar{\beta}_{i}=r_{i}^{(M)} \beta_{i}^{(M)}+r^{(i)} \beta^{(i)}
$$

With the above definitions, the side rotates an angle $\bar{\beta}_{i}$, the main element rotates $-\gamma_{i}^{(M)}$ relative to the side $\left(\gamma_{i}^{(M)}=\bar{\beta}_{i}-\beta_{i}^{(M)}\right)$ and the adjacent element rotates $+\gamma^{(i)}$ relative to the side. These definitions of the angles $\beta_{i}^{(M)}$ and $\beta^{(i)}$ are 'conceptual' as the direction $\mathbf{s}_{i}$ is not fixed in the space and it is not possible to measure $\beta_{i}^{(M)}$ and $\beta^{(i)}$ separately. In fact (30) is used and with it $\gamma_{i}^{(M)}$ and $\gamma^{(i)}$ are computed. However the variations of $\beta_{i}^{(M)}$ and $\beta^{(i)}$ can be indeed computed without problems.

Introducing now the definition of the relative rotation angles $\gamma_{i}^{(M)}$ at the redefined curvature (23) it yields

$$
\left[\begin{array}{c}
\kappa_{11} \\
\kappa_{22} \\
2 \kappa_{12}
\end{array}\right]=4 A \sum_{i=1}^{3} \frac{1}{l_{i}}\left[\begin{array}{c}
\left(L_{\prime_{1}}^{i}\right)^{2} \\
\left(L_{\prime_{2}}^{i}\right)^{2} \\
-2 L_{\prime_{1}}^{i} L_{\prime_{2}}^{i}
\end{array}\right] \gamma_{i}^{(M)}
$$

Note that these are in fact changes of curvature. This is so because in this definition relative rotations are used and they are null in the original configuration. This is not a disadvantage or limitation as in the implementation the strains are computed using the changes of curvature (see Equation (9)). Also note that the definition of the curvature in (39) depends strictly on the coordinates (relative positions) of the nodes in the patch. This leads to an objective definition that allows to satisfy exactly the rigid body motions.

Let us now compute the variations of the components of the change of curvature tensor. We need to compute:

$$
\delta\left(\lambda_{n_{i}}^{(M)} \gamma_{i}^{(M)}\right)=\lambda_{n_{i}}^{(M)} \delta \gamma_{i}^{(M)}+\gamma_{i}^{(M)} \delta \lambda_{n_{i}}^{(M)}
$$

Two contributions can be distinguished in the r.h.s. of Equation (40), the first term associated to the variation of relative angle is the most important, while the second term associated to a variation in the in-plane stretch may be disregarded (for linear problems it is effectively null).

The variation of the relative angle $\gamma_{i}^{(M)}$ results in

$$
\begin{aligned}
& \delta \gamma_{i}^{(M)}=r_{i}^{(M)} \delta\left(\Delta \phi_{i}\right)=r_{i}^{(M)}\left(\delta \beta^{(i)}-\delta \beta_{i}^{(M)}\right) \\
& \delta \gamma_{i}^{(M)}=r_{i}^{(M)}\left(\frac{1}{\lambda_{n}^{(i)}} \mathbf{t}^{(i)} \cdot \delta \boldsymbol{\varphi}_{\prime_{n}}^{(i)}+\frac{1}{\lambda_{n_{i}}^{(M)}} \mathbf{t}^{(M)} \cdot \delta \boldsymbol{\varphi}_{\prime_{n}}^{(M)}\right)
\end{aligned}
$$


where the variation of the gradient normal to the side $\boldsymbol{\varphi}_{{ }^{\prime} n}$, computed over the tangent plane to each triangle can be derived from the linear interpolation using the standard relations

$$
L_{{ }_{n}}^{I(M)}=\frac{c_{I}^{(M)}}{2^{o} A^{(M)}}
$$

where $c_{l}^{(M)}$ are the projections of the sides (original configuration) of the main triangle over the unit vector along the side ${ }^{o} \mathbf{s}_{i}$

$$
\begin{aligned}
& c_{1}^{(M)}=\left({ }^{o} \boldsymbol{\varphi}^{3}-{ }^{o} \boldsymbol{\varphi}^{2}\right) \cdot{ }^{o} \mathbf{s}_{i} \\
& c_{2}^{(M)}=\left({ }^{o} \boldsymbol{\varphi}^{1}-{ }^{o} \boldsymbol{\varphi}^{3}\right) \cdot{ }^{o} \mathbf{s}_{i} \\
& c_{3}^{(M)}=\left({ }^{o} \boldsymbol{\varphi}^{2}-{ }^{o} \boldsymbol{\varphi}^{1}\right) \cdot{ }^{o} \mathbf{s}_{i}
\end{aligned}
$$

so that

$$
\delta \boldsymbol{\varphi}_{n}^{(M)}=\frac{1}{2^{o} A^{(M)}}\left[c_{1}^{(M)}, c_{2}^{(M)}, c_{3}^{(M)}\right]\left[\begin{array}{c}
\delta \mathbf{u}^{1} \\
\delta \mathbf{u}^{2} \\
\delta \mathbf{u}^{3}
\end{array}\right]
$$

In the same way for the adjacent triangle $i$

$$
\delta \boldsymbol{\varphi}_{\prime n}^{(i)}=\frac{1}{2^{o} A^{(i)}}\left[c_{1}^{(i)}, c_{2}^{(i)}, c_{3}^{(i)}\right]\left[\begin{array}{c}
\delta \mathbf{u}^{(i) 1} \\
\delta \mathbf{u}^{(i) 2} \\
\delta \mathbf{u}^{(i) 3}
\end{array}\right]
$$

with $c_{l}^{(i)}$ the projections of the sides of the adjacent triangle over the unit vector along the side $-{ }^{o} \mathbf{s}_{i}$ and $\delta \mathbf{u}^{(i) j}$ the variation of the nodal displacement of node $j$ (local numeration over the element $i$ ). For instance, for the triangle adjacent to side 1 (see Figure 1(a))

$$
\begin{gathered}
c_{1}^{(1)}=-\left({ }^{o} \boldsymbol{\varphi}^{2}-{ }^{o} \boldsymbol{\varphi}^{3}\right) \cdot{ }^{o} \mathbf{s}_{1} \\
c_{2}^{(1)}=-\left({ }^{o} \boldsymbol{\varphi}^{4}-{ }^{o} \boldsymbol{\varphi}^{2}\right) \cdot{ }^{o} \mathbf{s}_{1} \\
c_{3}^{(1)}=-\left({ }^{o} \boldsymbol{\varphi}^{3}-{ }^{o} \boldsymbol{\varphi}^{4}\right) \cdot{ }^{o} \mathbf{s}_{1}
\end{gathered}
$$

With these definitions the variation of the change of angle $\Delta \phi_{i}$ results

$$
\begin{aligned}
\delta\left(\Delta \phi_{i}\right)= & \frac{1}{2^{o} A^{(M)} \lambda_{n_{i}}^{(M)}}\left[c_{1}^{(M)}, c_{2}^{(M)}, c_{3}^{(M)}\right]\left[\begin{array}{l}
\mathbf{t}^{(M)} \cdot \delta \mathbf{u}^{1} \\
\mathbf{t}^{(M)} \cdot \delta \mathbf{u}^{2} \\
\mathbf{t}^{(M)} \cdot \delta \mathbf{u}^{3}
\end{array}\right] \\
& +\frac{1}{2^{o} A^{(i)} \lambda_{n}^{(i)}}\left[c_{1}^{(i)}, c_{2}^{(i)}, c_{3}^{(i)}\right]\left[\begin{array}{l}
\mathbf{t}^{(i)} \cdot \delta \mathbf{u}^{(i) 1} \\
\mathbf{t}^{(i)} \cdot \delta \mathbf{u}^{(i) 2} \\
\mathbf{t}^{(i)} \cdot \delta \mathbf{u}^{(i) 3}
\end{array}\right]
\end{aligned}
$$


then Equation (40) can be written as

$$
\delta\left(\lambda_{n_{i}}^{(M)} \gamma_{i}^{(M)}\right)=r_{i}^{(M)}\left[\mathbf{t}^{(M)} \cdot \delta \boldsymbol{\varphi}_{{ }_{n}}^{(M)}+\mathbf{t}^{(i)} \cdot \delta \boldsymbol{\varphi}_{{ }_{n}}^{(i)}\right]+\gamma_{i}^{(M)} \delta \lambda_{n_{i}}^{(M)}
$$

where the influence of the second term will be disregarded for the computation of the variation. For linear problems above expression is exactly:

$$
\delta\left(\lambda_{n_{i}}^{(M)} \gamma_{i}^{(M)}\right)=r_{i}^{(M)}\left[\mathbf{t}^{(M)} \cdot \delta \boldsymbol{\varphi}_{{ }_{n}}^{(M)}+\mathbf{t}^{(i)} \cdot \delta \boldsymbol{\varphi}_{{ }_{n}}^{(i)}\right]
$$

Substituting Equation (50) into (24) allows to compute the variation of the change of curvature for the element.

\section{BRANCHING SHELLS}

The case considered in the previous section can be seen as a particular case (the simplest one) of a multi-surface intersection (branching). In a general case there will be $n$ surfaces (elements) sharing a side (s). To simplify the notation, let us assume the common side to be the first side (opposite to first node) of each element neighbour to this branching. Also let us assume a common orientation of the side for all the elements (see Figure 4). Let us denote by $J$ and $K$ (fixed) the nodes defining the side (in that order) and by $i$ the remaining node of each (generic) element $i$ at the branching.

In the original configuration the tangent plane to each triangle $i$ is defined by the unit vector along the side ${ }^{o} \mathbf{s}$ (identically oriented in all the triangles) and the (outward) normal to the side

$$
{ }^{o} \mathbf{n}_{i}={ }^{o} \mathbf{s} \times{ }^{o} \mathbf{t}^{(i)}
$$

written in terms of the side direction and the element normal ${ }^{o} \mathbf{t}^{(i)}$. The $n$ normal vectors ${ }^{o} \mathbf{t}^{(i)}$ allow to define a set of $(n-1)$ independent angles between the different elements $(i=1, \ldots, n-1)$

$$
\begin{aligned}
& \cos { }^{o} \phi_{i}={ }^{o} \mathbf{t}^{(i)} \cdot{ }^{o} \mathbf{t}^{(i+1)}={ }^{o} \mathbf{n}^{(i)} \cdot{ }^{o} \mathbf{n}^{(i+1)} \\
& \sin { }^{o} \phi_{i}={ }^{o} \mathbf{n}^{(i)} \cdot{ }^{o} \mathbf{t}^{(i+1)}=-{ }^{o} \mathbf{t}^{(i)} \cdot{ }^{o} \mathbf{n}^{(i+1)}
\end{aligned}
$$
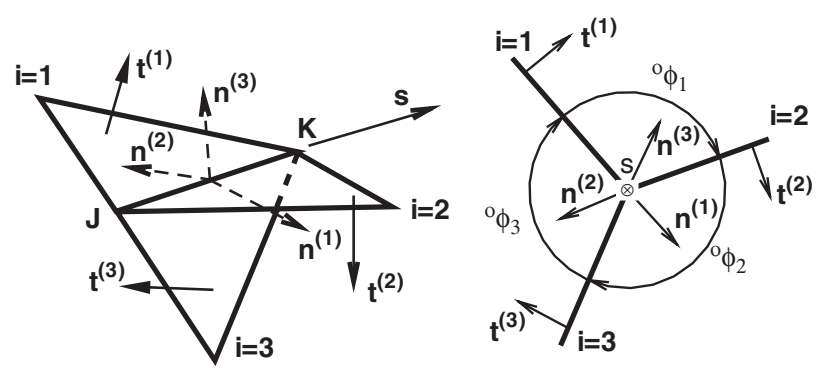

Figure 4. Surface branching. 
that can be completed with the angle between the last and the first elements (unnecessary due to its dependence with the others)

$$
\phi_{n}=2 \pi-\bmod \left[\sum_{i=1}^{n-1} \phi_{i}, 2 \pi\right]
$$

In the deformed configuration the actual common unit vector $\mathbf{s}$ will be:

$$
\mathbf{s}=\frac{\varphi^{K}-\varphi^{J}}{\left\|\varphi^{K}-\varphi^{J}\right\|}=\frac{\varphi^{K}-\varphi^{J}}{l_{s}}=\frac{\varphi^{K}-\varphi^{J}}{{ }^{o} l_{s} \lambda_{s}}
$$

(this fibre stretches $\lambda_{s}$ respect to the original configuration) that together with the new normal at each element $\mathbf{t}^{(i)}$ allows to compute the new outward normal at each element

$$
\mathbf{n}^{(i)}=\mathbf{s} \times \mathbf{t}^{(i)}
$$

and with $\mathbf{t}^{(i)}$ 's and $\mathbf{n}^{(i)}$ 's the new set of $n-1$ angles will be

$$
\begin{aligned}
& \cos \phi_{i}=\mathbf{t}^{(i)} \cdot \mathbf{t}^{(i+1)}=\mathbf{n}^{(i)} \cdot \mathbf{n}^{(i+1)} \\
& \sin \phi_{i}=\mathbf{n}^{(i)} \cdot \mathbf{t}^{(i+1)}=-\mathbf{t}^{(i)} \cdot \mathbf{n}^{(i+1)}
\end{aligned}
$$

that have changed with respect to the original ones by

$$
\Delta \phi_{i}=\phi_{i}-{ }^{o} \phi_{i}, \quad i=1, n-1
$$

As the direction $\mathbf{s}$ rotates in space, there is not a fixed reference to measure the angle $\beta_{i}$ rotated by each element. It can be therefore assumed that the first element does not rotate $\left(\beta^{(1)}=0\right)$ and compute the rest of the rotations using the first element as the reference. This leads to

$$
\beta^{(i)}=\sum_{m=1}^{i-1} \Delta \phi_{m}
$$

In a similar way the rotation of the side, expression (38), is evaluated as the weighted average

$$
\bar{\beta}=\frac{1}{\sum_{l=1}^{n} R^{(l)}} \sum_{i=1}^{n} R^{(i)} \beta^{(i)}=\sum_{i=1}^{n} r^{(i)} \beta^{(i)}
$$

where similarly to (36) a relative stiffness has been introduced

$$
r^{(i)}=\frac{R^{(i)}}{\sum_{l=1}^{n} R^{(l)}}
$$

We denote now with $\gamma_{i}$ the difference between the (average) angle rotated by the side $\bar{\beta}$ and the angle rotated by the element $\beta^{(i)}$

$$
\gamma_{i}=\bar{\beta}-\beta^{(i)}
$$

The above expression (61) can be explicitly written for the case $n=2$ :

$$
\left[\begin{array}{l}
\gamma_{1} \\
\gamma_{2}
\end{array}\right]=\left[\begin{array}{c}
\bar{\beta} \\
\bar{\beta}-\Delta \phi_{1}
\end{array}\right]=\left[\begin{array}{c}
1-r^{(1)} \\
-r^{(1)}
\end{array}\right] \Delta \phi_{1}
$$


for the case $n=3$

$$
\left[\begin{array}{l}
\gamma_{1} \\
\gamma_{2} \\
\gamma_{3}
\end{array}\right]=\left[\begin{array}{c}
\bar{\beta} \\
\bar{\beta}-\Delta \phi_{1} \\
\bar{\beta}-\Delta \phi_{1}-\Delta \phi_{2}
\end{array}\right]=\left[\begin{array}{cc}
1-r^{(1)} & 1-r^{(1)}-r^{(2)} \\
-r^{(1)} & 1-r^{(1)}-r^{(2)} \\
-r^{(1)} & -r^{(1)}-r^{(2)}
\end{array}\right]\left[\begin{array}{c}
\Delta \phi_{1} \\
\Delta \phi_{2}
\end{array}\right]
$$

For a general case it can also be expressed as:

$$
\begin{aligned}
\Gamma & =\mathbf{C}_{n \times(n-1)} \Delta \phi_{n-1} \\
\gamma_{i} & =C_{i m} \Delta \phi_{m}
\end{aligned}
$$

From the definition of $\gamma_{i}$ (see Figure 3 and Equation (61)), the gradient in the outward normal to the side direction $\overline{\boldsymbol{\varphi}}_{{ }_{n}}^{(i)}$ at each one of the concurrent elements, can be resolved in its components over a local triad as

$$
\begin{aligned}
\overline{\boldsymbol{\varphi}}_{\prime n}^{(i)} & =\left(\overline{\boldsymbol{\varphi}}_{\prime n}^{(i)} \cdot \mathbf{n}^{(i)}\right) \mathbf{n}^{(i)}+\left(\overline{\boldsymbol{\varphi}}_{\prime}^{(i)} \cdot \mathbf{s}\right) \mathbf{s}+\left(\overline{\boldsymbol{\varphi}}_{\prime n}^{(i)} \cdot \mathbf{t}^{(i)}\right) \mathbf{t}^{(i)} \\
& =\lambda_{n}^{(i)} \cos \gamma_{i} \mathbf{n}^{(i)}+a_{n s} \mathbf{s}+\lambda_{n}^{(i)} \sin \gamma_{i} \mathbf{t}^{(i)}
\end{aligned}
$$

The component along the direction normal to the element plane is

$$
\overline{\boldsymbol{\varphi}}_{n}^{(i)} \cdot \mathbf{t}^{(i)}=\lambda_{n}^{(i)} \sin \gamma_{i} \simeq \lambda_{n}^{(i)} \gamma_{i}
$$

Replacing (66) into (23) allows to complete the evaluation of the curvature at each of the elements neighbours to the side.

To evaluate the curvature variation (24) it is necessary to compute the variation of the normal to the side component of the gradient. This is composed of two parts:

$$
\begin{aligned}
\delta\left(\overline{\boldsymbol{\varphi}}_{\prime n}^{(i)} \cdot \mathbf{t}^{(i)}\right) & =\delta\left(\lambda_{n}^{(i)} \gamma_{i}\right) \\
& =\lambda_{n}^{(i)} \delta \gamma_{i}+\gamma_{i} \delta \lambda_{n}^{(i)}
\end{aligned}
$$

where again the important term is the first.

The variation of the angle $\gamma_{i}$ results from observing Equations (63) and (56)

$$
\begin{aligned}
\delta \gamma_{i} & =C_{i m} \delta \phi_{j}, \quad i=1, n, \quad m=1, n-1 \\
& =C_{i m}\left[\frac{1}{\lambda_{n}^{(m)}} \mathbf{t}^{(m)} \cdot \delta \boldsymbol{\varphi}_{n}^{(m)}+\frac{1}{\lambda_{n}^{(m+1)}} \mathbf{t}^{(m+1)} \cdot \delta \boldsymbol{\varphi}_{n}^{(m+1)}\right]
\end{aligned}
$$

where $\delta \boldsymbol{\varphi}_{n}^{(m)}$ are the variations of the normal gradients computed over the tangent plane of each triangle. In a standard way:

$$
\delta \boldsymbol{\varphi}_{\prime_{n}}^{(m)}=\frac{1}{2 A^{(m) o}}\left[c_{m}^{(m)}, c_{J}^{(m)}, c_{K}^{(m)}\right]\left[\begin{array}{c}
\delta \mathbf{u}^{m} \\
\delta \mathbf{u}^{J} \\
\delta \mathbf{u}^{K}
\end{array}\right]
$$


with $c_{l}^{(m)}$ the projections of each element side over the unit vector along the side ${ }^{o} \mathbf{S}$

$$
\begin{aligned}
c_{m}^{(m)} & =\left({ }^{o} \boldsymbol{\varphi}^{K}-{ }^{o} \boldsymbol{\varphi}^{J}\right) \cdot{ }^{o} \mathbf{s}={ }^{o} l_{s} \quad \text { (equal for all elements) } \\
c_{J}^{(m)} & =\left({ }^{o} \boldsymbol{\varphi}^{m}-{ }^{o} \boldsymbol{\varphi}^{K}\right) \cdot{ }^{o} \mathbf{s} \\
c_{K}^{(m)} & =\left({ }^{o} \boldsymbol{\varphi}^{J}-{ }^{o} \boldsymbol{\varphi}^{m}\right) \cdot{ }^{o} \mathbf{s}
\end{aligned}
$$

With this information, the variation of the angle $\gamma_{i}$ results

$$
\begin{aligned}
\delta \gamma_{i}= & \sum_{m=1}^{n-1} C_{i m}\left\{\frac{1}{2^{o} A^{(m)} \lambda_{n}^{(m)}}\left[c_{m}^{(m)}, c_{J}^{(m)}, c_{K}^{(m)}\right]\left[\begin{array}{c}
\mathbf{t}^{(m)} \cdot \delta \mathbf{u}^{m} \\
\mathbf{t}^{(m)} \cdot \delta \mathbf{u}^{J} \\
\mathbf{t}^{(m)} \cdot \delta \mathbf{u}^{K}
\end{array}\right]\right. \\
& \left.+\frac{1}{2^{o} A^{(m+1)} \lambda_{n}^{(m+1)}}\left[c_{m+1}^{(m+1)}, c_{J}^{(m+1)}, c_{K}^{(m+1)}\right]\left[\begin{array}{c}
\mathbf{t}^{(m+1)} \cdot \delta \mathbf{u}^{m+1} \\
\mathbf{t}^{(m+1)} \cdot \delta \mathbf{u}^{J} \\
\mathbf{t}^{(m+1)} \cdot \delta \mathbf{u}^{K}
\end{array}\right]\right\}
\end{aligned}
$$

Recalling (61), the above expression can be simplified to

$$
\begin{aligned}
\delta \gamma_{i} & =\delta \bar{\beta}-\delta \beta^{(i)} \\
& =\left[\frac{1}{\lambda_{n}^{(i)}} \mathbf{t}^{(i)} \cdot \delta \boldsymbol{\varphi}_{{ }_{n}}^{(i)}-\sum_{m=1}^{n} r^{(m)} \frac{1}{\lambda_{n}^{(m)}} \mathbf{t}^{(m)} \cdot \delta \boldsymbol{\varphi}_{n}^{(m)}\right]
\end{aligned}
$$

with this result Equation (40) is

$$
\delta\left(\lambda_{n}^{(i)} \gamma_{i}\right)=\left[\mathbf{t}^{(i)} \cdot \delta \boldsymbol{\varphi}_{n}^{(i)}-\lambda_{n}^{(i)} \sum_{m=1}^{n} r^{(m)} \frac{1}{\lambda_{n}^{(m)}} \mathbf{t}^{(m)} \cdot \delta \boldsymbol{\varphi}_{n}^{(m)}\right]+\gamma_{i} \delta \lambda_{n}^{(i)}
$$

Note that because $\mathbf{s}$ is chosen as the reference direction, the variation of the rotation of each element is:

$$
\delta \beta^{(i)}=-\mathbf{t}^{(i)} \cdot \delta \boldsymbol{\varphi}_{n}^{(i)}
$$

For linear problems, the above expression simplifies to:

$$
\delta\left(\lambda_{n}^{(i)} \gamma_{i}\right)=\left[\mathbf{t}^{(i)} \cdot \delta \boldsymbol{\varphi}_{n}^{(i)}-\sum_{m=1}^{n} r^{(m)} \mathbf{t}^{(m)} \cdot \delta \boldsymbol{\varphi}_{n}^{(m)}\right]
$$

Expression (74) allows to compute the curvature variations at any element using Equation (24), where the form of $\delta\left(\lambda_{n}^{(i)} \gamma_{i}\right)$ over each side of an element can be: (a) Equation (50) if the side is part of a unique surface (smooth or not) or (b) Equation (76) if it is a branching line. In the usual notation of the finite element method, the curvature variation can be expressed as

$$
\delta \mathbf{\kappa}=\mathbf{B}_{b} \delta \mathbf{u}^{p}
$$


It must be noted that this variation depends on the nodes of all the elements that share a side with the element where such variation is computed. Then, the vector $\delta \mathbf{u}^{p}$ includes, in an element without branching, the six nodes indicated in the patch of Figure 1. When an element has a branching side, the number of nodes included in $\delta \mathbf{u}^{p}$ is four plus the number of elements that share the side. Naturally the size of the elemental stiffness matrix depends on the number of unknowns included in vector $\delta \mathbf{u}^{p}$.

\section{COMPUTATION OF THE ANGLE VARIATION FOR THE EBST ELEMENT}

In Reference [15] a similar element to the BST element was developed under the framework of an assumed strain method. The main advantage of this new rotation-free shell element (termed EBST) is that its membrane performance is similar to the linear strain triangle, in contrast with the BST that has a constant strain membrane behaviour. To apply the developments of the previous sections to the EBST element, let us first recall how the curvatures are computed for this element.

The numerical definition of the curvatures follows the same expression used above (17)

$$
\left[\begin{array}{c}
\kappa_{11} \\
\kappa_{22} \\
2 \kappa_{12}
\end{array}\right]=\frac{-1}{{ }^{o} A} \sum_{i=1}^{3}{ }^{o} l_{i}\left[\begin{array}{cc}
{ }^{o} n_{1} & 0 \\
0 & { }^{o} n_{2} \\
{ }^{o} n_{2} & { }^{o} n_{1}
\end{array}\right]_{i}\left[\begin{array}{l}
\boldsymbol{\varphi}_{1} \cdot \mathbf{t}^{(M)} \\
\boldsymbol{\varphi}_{\prime 2} \cdot \mathbf{t}^{(M)}
\end{array}\right]_{i}
$$

The difference is how the gradient at the mid-side points is computed. In the EBST element a quadratic interpolation of the geometry is used based on the six nodes belonging to the standard patch (see Figure 1(b))

$$
\boldsymbol{\varphi}=\sum_{I=1}^{6} N^{I}\left(\eta_{1}, \eta_{2}\right) \boldsymbol{\varphi}^{I}
$$

with $\left(\eta_{3}=1-\eta_{1}-\eta_{2}\right)$

$$
\begin{array}{ll}
N^{1}=\eta_{3}+\eta_{1} \eta_{2}, & N^{4}=\frac{\eta_{3}}{2}\left(\eta_{3}-1\right) \\
N^{2}=\eta_{1}+\eta_{2} \eta_{3}, & N^{5}=\frac{\eta_{1}}{2}\left(\eta_{1}-1\right) \\
N^{3}=\eta_{2}+\eta_{3} \eta_{1}, & N^{6}=\frac{\eta_{2}}{2}\left(\eta_{2}-1\right)
\end{array}
$$

As it is shown in Reference [15], at each mid-side point the gradient depends exclusively on the co-ordinates of the four nodes associated to the two elements adjacent to the side $i$ (the upper-index surrounded by round brackets indicates now evaluated at the mid-point of side $i$ ), i.e.

$$
\left[\begin{array}{l}
\boldsymbol{\varphi}_{\prime 1} \\
\boldsymbol{\varphi}_{22}
\end{array}\right]^{(i)}=\left[\begin{array}{llll}
N_{1}^{1} & N_{11}^{2} & N_{11}^{3} & N_{\prime 1}^{i+3} \\
N_{\prime 2}^{1} & N_{\prime_{2}}^{2} & N_{/ 2}^{3} & N_{/ 2}^{i+3}
\end{array}\right]^{(i)}\left[\begin{array}{c}
\varphi^{1} \\
\varphi^{2} \\
\varphi^{3} \\
\varphi^{i+3}
\end{array}\right]
$$


The EBST element is also non-conforming, but a stronger continuity of the normal is imposed than in the BST element.

Similarly to the BST when the gradient is projected on the two local directions $\left({ }^{o} \mathbf{n},{ }^{o} \mathbf{s}\right)$ at the side, the product

$$
\boldsymbol{\varphi}_{i s}^{(i)} \cdot \mathbf{t}^{(M)}=0
$$

is null because

$$
\varphi_{{ }_{s}}^{(i)}=\frac{1}{{ }^{o} l_{s}}\left(\varphi^{K}-\varphi^{J}\right)
$$

Using Equation (20) an expression almost identical to Equation (21) is obtained (see Reference [15] for details)

$$
\left[\begin{array}{c}
\kappa_{11} \\
\kappa_{22} \\
2 \kappa_{12}
\end{array}\right]=-4^{o} A \sum_{i=1}^{3} \frac{1}{o} l_{i}\left[\begin{array}{c}
\left(L_{\prime_{1}}^{i}\right)^{2} \\
\left(L_{\prime_{2}}^{i}\right)^{2} \\
-2 L_{{ }_{1}}^{i} L_{\prime_{2}}^{i}
\end{array}\right]\left(\boldsymbol{\varphi}_{\prime_{n}}^{(i)} \cdot \mathbf{t}^{(M)}\right)
$$

Note that now $\boldsymbol{\varphi}_{/ n}^{(i)}$ is not the gradient computed at the adjacent element in the outward normal direction at its boundary, but the gradient computed at the mid-side point in the outward normal direction of the main element boundary. This leads to two differences with expression (21): the $\frac{1}{2}$ of the averaging disappears and the sign is not changed. Using consistently the angle between normals instead of the angle sine, the projection of the gradient normal to the boundary can be rewritten as

$$
\begin{aligned}
\boldsymbol{\varphi}_{{ }_{n}}^{(i)} \cdot \mathbf{t}^{(M)} & =-\lambda_{n}^{(i)} \sin \left(\mathbf{n}^{(i)} \cdot \mathbf{t}^{(M)}\right)=\lambda_{n}^{(i)} \sin \gamma_{i} \\
& \cong-\lambda_{n}^{(i)} \gamma_{i} \equiv-\lambda_{n_{i}}^{(M)} \gamma_{i}
\end{aligned}
$$

that allows to re-obtain Equation (23). In this last expression the angle $\gamma_{i}$ can be interpreted as the angle formed by $\boldsymbol{\varphi}_{{ }_{n}}^{(i)}$ with the tangent plane at the main triangle. When the surfaces are initially curved $\gamma_{i}$ is not null and in contrast with the BST element there exists initial curvatures (see Equation (39)). It is also possible to reinterpret $\gamma_{i}$ as the change of angle between the original and deformed configurations and disregard the influence of the initial curvatures in the computation of the deformation gradient at points outside the middle surface, similarly to the BST element. Finally, it is possible to consider the different bending stiffness of the adjacent elements and to apply the factor $r_{i}^{(M)}$ (see Equation (36a)) to the angles $\gamma_{i}$ when the curvature is computed.

The second aspect is the computation of the curvature variation for the weak form of the momentum equations. To obtain Equation (24) the variation of the component of the normal gradient over the element normal is needed

$$
\delta\left(\lambda_{n_{i}}^{(M)} \gamma_{i}\right)=\lambda_{n_{i}}^{(M)} \delta \gamma_{i}+\gamma_{i} \delta \lambda_{n_{i}}^{(M)}
$$


The variation of the angle $\gamma_{i}$ results (with $\mathbf{t}^{(i)}$ being the normal to the tangent plane at the side $i$ )

$$
\delta \gamma_{i}=\frac{1}{\lambda_{n_{i}}^{(M)}} \mathbf{t}^{(i)} \cdot \delta \boldsymbol{\varphi}_{{ }_{n}}^{(i)}
$$

where the variation of the gradient normal to the side $\boldsymbol{\varphi}_{{ }_{n}}^{(i)}$ computed at the mid-side point is:

$$
\delta \boldsymbol{\varphi}_{\prime n}^{(i)}=\left[\begin{array}{llll}
N_{\prime n}^{1} & N_{\prime n}^{2} & N_{\prime n}^{3} & N_{\prime n}^{i+3}
\end{array}\right]^{(i)}\left[\begin{array}{c}
\delta \mathbf{u}^{1} \\
\delta \mathbf{u}^{2} \\
\delta \mathbf{u}^{3} \\
\delta \mathbf{u}^{i+3}
\end{array}\right]
$$

where $N_{l_{n}}^{J}$ are the derivatives of the shape functions (80) in the direction normal to the boundary.

With the above definitions the variation of the angle $\gamma_{i}$ is

$$
\delta\left(\gamma_{i}\right)=\frac{1}{\lambda_{n_{i}}^{(M)}}\left[\begin{array}{llll}
N_{\prime n}^{1} & N_{\prime n}^{2} & N_{\prime n}^{3} & N_{\prime n}^{i+3}
\end{array}\right]^{(i)}\left[\begin{array}{c}
\mathbf{t}^{(i)} \cdot \delta \mathbf{u}^{1} \\
\mathbf{t}^{(i)} \cdot \delta \mathbf{u}^{2} \\
\mathbf{t}^{(i)} \cdot \delta \mathbf{u}^{3} \\
\mathbf{t}^{(i)} \cdot \delta \mathbf{u}^{I+3}
\end{array}\right]
$$

The quadratic interpolation used in the EBST element assumes (as for the BST case) that the surface is smooth and that an adequate discretization is chosen which is capable of adequately model the curvatures (the initial ones or those produced during the deformation). Elements with a side part of a kink or a branching may be treated as for the BST element in the previous sections.

\section{MEMBRANE BEHAVIOUR AND STIFFNESS MATRIX}

With the aim to make this work self-contained, the membrane formulation and the computation of the stiffness matrix are described next.

The membrane deformation may be expressed in terms of the Green-Lagrange (14) strain tensor or in terms of the metric tensor over the middle surface (6). The former may be written in the usual notation of the FEM as

$$
\left[\begin{array}{c}
E_{11} \\
E_{22} \\
2 E_{12}
\end{array}\right]=\frac{1}{2}\left[\begin{array}{c}
a_{11}-1 \\
a_{22}-1 \\
2 a_{12}
\end{array}\right]=\frac{1}{2}\left[\begin{array}{c}
\boldsymbol{\varphi}_{\prime 1} \cdot \boldsymbol{\varphi}_{\prime}^{\prime}-1 \\
\boldsymbol{\varphi}_{2} \cdot \boldsymbol{\varphi}_{\prime 2}-1 \\
2 \boldsymbol{\varphi}_{\prime} \cdot \boldsymbol{\varphi}_{2}
\end{array}\right]
$$


while the virtual strains can be directly obtained as

$$
\delta\left[\begin{array}{c}
E_{11} \\
E_{22} \\
2 E_{12}
\end{array}\right]=\frac{1}{2}\left[\begin{array}{c}
\delta a_{11} \\
\delta a_{22} \\
2 \delta a_{12}
\end{array}\right]=\left[\begin{array}{c}
\boldsymbol{\varphi}_{11} \cdot \delta \boldsymbol{\varphi}_{\prime}^{\prime} \\
\boldsymbol{\varphi}_{22} \cdot \delta \boldsymbol{\varphi}_{2}^{\prime} \\
\boldsymbol{\varphi}_{\prime 1} \cdot \delta \boldsymbol{\varphi}_{\prime 2}+\delta \boldsymbol{\varphi}_{\prime}^{\prime} \cdot \boldsymbol{\varphi}_{\prime 2}
\end{array}\right]
$$

In the BST element, the membrane strains are computed using a linear interpolation of the middle surface from the three nodes of the main element (16), leading to the well-known constant strain triangle. The matrix membrane strain $\mathbf{B}_{m}$ relating the strain variations with the displacement variations can be expressed as

$$
\begin{aligned}
\delta\left[\begin{array}{c}
E_{11} \\
E_{22} \\
2 E_{12}
\end{array}\right] & =\left[\begin{array}{cc}
\boldsymbol{\varphi}_{\prime 1}^{\mathrm{T}} & \mathbf{0}_{3 \times 1}^{\mathrm{T}} \\
\mathbf{0}_{3 \times 1}^{\mathrm{T}} & \boldsymbol{\varphi}_{\prime 2}^{\mathrm{T}} \\
\boldsymbol{\varphi}_{\prime 2}^{\mathrm{T}} & \boldsymbol{\varphi}_{\prime 1}^{\mathrm{T}}
\end{array}\right]\left[\begin{array}{ccc}
L_{\prime 1}^{1} & L_{\prime 1}^{2} & L_{\prime 1}^{3} \\
L_{\prime 2}^{1} & L_{\prime 2}^{2} & L_{\prime 2}^{3}
\end{array}\right]\left[\begin{array}{c}
\delta \mathbf{u}^{1} \\
\delta \mathbf{u}^{2} \\
\delta \mathbf{u}^{3}
\end{array}\right] \\
& =\mathbf{B}_{m} \delta \mathbf{u}
\end{aligned}
$$

where $L_{l j}^{i}$ are the shape function given in Equation (20). In this case the array $\delta \mathbf{u}$ include only the displacement of the three nodes of the main triangle $(M)$.

For the EBST element the geometry of the middle surface is defined by the quadratic approximation (79). The deformation gradient computed at each mid-side point (81), used above for the evaluation of the curvatures (82), is now also used to compute the metric tensor $a_{\alpha \beta}^{(i)}$ at the same points. These metric tensors are averaged at the element centre

$$
\bar{a}_{\alpha \beta}=\frac{1}{3} \sum_{i=1}^{3} a_{\alpha \beta}^{(i)}
$$

and with this average $\bar{a}_{\alpha \beta}$ the Green-Lagrange strain tensor (14) is computed. The variation of this tensor results from the average of:

$$
\begin{aligned}
\delta\left[\begin{array}{c}
a_{11} \\
a_{22} \\
2 a_{12}
\end{array}\right]^{(i)}=\delta\left[\begin{array}{c}
E_{11} \\
E_{22} \\
2 E_{12}
\end{array}\right]^{(i)}=\left[\begin{array}{cc}
\boldsymbol{\varphi}_{\prime 1}^{\mathrm{T}} & \mathbf{0}_{3 \times 1}^{\mathrm{T}} \\
\mathbf{0}_{3 \times 1}^{\mathrm{T}} & \boldsymbol{\varphi}_{\prime 2}^{\mathrm{T}} \\
\boldsymbol{\varphi}_{\prime 2}^{\mathrm{T}} & \boldsymbol{\varphi}_{\prime 1}^{\mathrm{T}}
\end{array}\right]^{(i)}\left[\begin{array}{llll}
N_{\prime 1}^{1} & N_{\prime 1}^{2} & N_{1}^{3} & N_{\prime 1}^{i+3} \\
N_{\prime 2}^{1} & N_{\prime 2}^{2} & N_{\prime 2}^{3} & N_{\prime 2}^{i+3}
\end{array}\right]\left[\begin{array}{c}
\delta \mathbf{u}^{1} \\
\delta \mathbf{u}^{2} \\
\delta \mathbf{u}^{3} \\
\delta \mathbf{u}^{i+3}
\end{array}\right] \\
=\mathbf{B}_{m}^{(i)} \delta \mathbf{u}^{(i)}
\end{aligned}
$$

In this quadratic approximation there are, of course, contributions from the six nodes of the four triangles in the patch.

The quadratic interpolation is sensitive to the position of the six nodes of the patch. Because of this, for the computation of the membrane strain, it is necessary to limit the allowable angle between the main element and the adjacent ones. This is of relevant importance in problems with very low or null bending stiffness (membranes or quasi-membranes) where wrinkles and very marked folds may occur. A possible solution is to perform at each side a gradual change from the quadratic approach 
(81) to the linear approach (20) as a function of the angle between the normals at the adjacent elements. Besides that, for elements where the adjacent element does not exist (shell boundary), or with a side over a kink or a branching region, good results have been obtained considering directly the metric tensor obtained from Equation (20) as the side contribution to the average (91).

The computation of the element stiffness matrix is standard. The material part is

$$
\mathbf{K}^{M}=\int_{A^{(M)}} \mathbf{B}^{\mathrm{T}} \mathbf{C B} \mathrm{d} A
$$

where the matrix $\mathbf{B}=\mathbf{B}_{m}+\mathbf{B}_{b}$ includes the corresponding $\mathbf{B}$ matrices according to the approximation chosen for the membrane and bending parts and the mesh topology. Matrix $\mathbf{C}$ depends on the constitutive material model and can be obtained integrating (numerically or analytically if possible) through the thickness. All the $\mathbf{B}$ matrices are constant and one integration point per element is enough for a correct numerical integration.

The disadvantage of the present formulation when compared with a standard 6 parameter theory is that the computer implementation is more involved as the element stiffness matrix depends on the mesh topology, not only for the elements adjacent to a branching but also for elements adjacent to the boundary. Note however that most of the elements will be part of four-element standard patches.

With respect to the geometric stiffness matrix, numerical experiments have shown that the contributions due to bending $\mathbf{K}_{b}^{G}$, that are rather complex to be computed exactly, can be disregarded. The contributions due to membrane forces $\mathbf{K}_{m}^{G}$ can be computed as follows:

$$
\delta \mathbf{u}^{\mathrm{T}} \mathbf{K}_{m}^{G} \Delta \mathbf{u}=\int_{A^{(M)}} \frac{\partial}{\partial \mathbf{u}}\left(\delta \boldsymbol{\varepsilon}^{\mathrm{T}} \mathbf{N}\right) \Delta \mathbf{u} \mathrm{d} A
$$

for the element BST they are standard

$$
\begin{aligned}
& \delta \mathbf{u}^{\mathrm{T}} \mathbf{K}_{m}^{G} \Delta \mathbf{u}=A^{(M)} \sum_{I=1}^{3} \sum_{J=1}^{3} L_{\prime_{1}}^{I} L_{{ }_{1}}^{J} N_{11}+L_{{ }^{2}}^{I} L_{{ }^{2}}^{J} N_{22}+\left(L_{{ }_{1}}^{I} L_{\prime_{2}}^{J}+L_{\iota_{2}}^{I} L_{\iota_{1}}^{J}\right) N_{12} \delta \mathbf{u}^{J} \cdot \Delta \mathbf{u}^{I} \\
& =A^{(M)} \sum_{I=1}^{3} \sum_{J=1}^{3}\left\{\delta \mathbf{u}^{I}\left[\begin{array}{ll}
L_{\prime 1}^{I} & L_{\prime 2}^{I}
\end{array}\right]\left[\begin{array}{ll}
N_{11} & N_{12} \\
N_{21} & N_{22}
\end{array}\right]\left[\begin{array}{c}
L_{\prime 1}^{J} \\
L_{\prime 2}^{J}
\end{array}\right] \Delta \mathbf{u}^{J}\right\}
\end{aligned}
$$

where $N_{\alpha \beta}$ are the second Piola-Kirchhoff stress Cartesian components (11a) integrated through the thickness.

For the element EBST, the membrane geometric stiffness can be expressed as the sum of the contributions from the three element sides, i.e. [15]

$$
\begin{aligned}
& \delta \mathbf{u}^{\mathrm{T}} \mathbf{K}_{m}^{G} \Delta \mathbf{u}=\frac{A^{(M)}}{3} \sum_{K=1}^{3} \sum_{I=1}^{4} \sum_{J=1}^{4}\left[N_{\prime_{1}}^{(K) I} N_{\prime_{1}}^{(K) J} N_{11}+N_{\prime_{2}}^{(K) I} N_{\prime_{2}}^{(K) J} N_{22}\right. \\
& \left.+\left(N_{11}^{(K) I} N_{\prime 2}^{(K) J}+N_{\prime 2}^{(K) I} N_{\prime 1}^{(K) J}\right) N_{12}\right] \delta \mathbf{u}^{(K) J} \cdot \Delta \mathbf{u}^{(K) I} \\
& =\frac{A^{(M)}}{3} \sum_{K=1}^{3} \sum_{I=1}^{4} \sum_{J=1}^{4}\left\{\delta \mathbf{u}^{I}\left[\begin{array}{ll}
N_{\prime}^{I} & N_{\prime 2}^{I}
\end{array}\right]\left[\begin{array}{ll}
N_{11} & N_{12} \\
N_{21} & N_{22}
\end{array}\right]\left[\begin{array}{l}
N_{\prime 1}^{J} \\
N_{\prime 2}^{J}
\end{array}\right] \Delta \mathbf{u}^{J}\right\}^{(K)}
\end{aligned}
$$


where the sum in $K=1, \ldots, 3$ is over the mid-side points and the sums in $I, J=1, \ldots, 4$ are over the four nodes that have contributions to the gradient at those points.

As mentioned above (see Equation (3)) the thickness stretch is constant across the thickness. A usual approach for large strain analysis, also adopted here, is to assume an isochoric behaviour, so that the thickness update is performed on a geometrical basis only. This has negligible consequences no only when elastic strains are small, as is the case for metals where plastic strain are normally assumed isochoric, but also for rubbers where large elastic strains are present but the whole behaviour is normally assumed isochoric.

\section{NUMERICAL EXAMPLES}

In this section several examples are presented to assess the performance of the rotation-free shell element presented for non-smooth and branching surfaces. The element used in the simulations, denoted as branching basic shell triangle (BBST), uses for the membrane and bending parts those corresponding to the EBST element where the surface is smooth. In all cases the residual forces and stiffness matrices are computed using one integration point over the element area and four points through the thickness. All the results shown have been obtained with an implicit program for static elastic problems developed by the first author. An exception is the last example that includes contact and elastic-plastic behaviour, that was solved using a code with explicit integration in time of the momentum equations [23].

\subsection{Twisted beam}

This first example is a widely used benchmark in linear analysis to assess the sensitivity of shell elements to initial warping. The fixed geometrical (non-dimensional) parameters of this cantilever beam (see Figure 5) are: length $L=12$, width $b=1.1$, angle of twist $90^{\circ}$, Poisson's ratio $v=0.3$ and Young Modulus $E=2.9 \times 10^{7}$. Two values were considered for the thickness, a thick case $h=0.32$ and a thin case $h=0.032$. In the first case the Jacobian has an important variation across the thickness. A distributed load is applied at the free end ( 1 for the thick case and 0.001 for the thin case), Figure 5 shows the load applied in the out-of-plane direction. The in-plane load case was also considered here. Two structured meshes were used, a coarse one with 48 elements $(2 \times 12$ divisions as shown in the figure $)$ and a fine mesh with 768 elements $(8 \times 48$ divisions $)$.

Table I presents the linear results obtained with BBST/EBST element showing the very good performance obtained with the coarse mesh. The exact values and the results reported in Reference [18] using the 'degenerated solid approach' on the coarse mesh are also included for comparison.

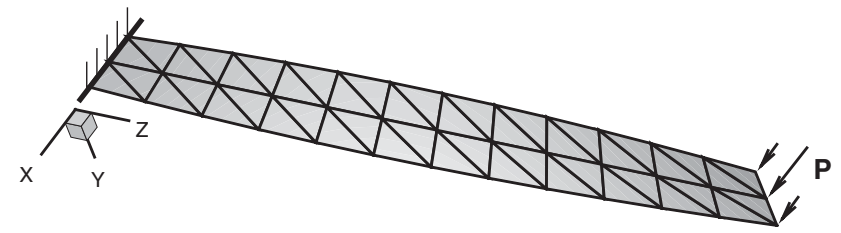

Figure 5. Twisted beam. 
Table I. Twisted beam.

\begin{tabular}{lccc}
\hline & Thick, in-plane & Thick, out-of-plane & Thin, out-of plane \\
\hline Coarse mesh & 0.005449 & 0.001892 & 0.001261 \\
Fine mesh & 0.005373 & 0.001747 & 0.001293 \\
Reference [18], coarse & & 0.001887 & 0.001281 \\
Exact & 0.005424 & 0.001754 & 0.001294 \\
\hline
\end{tabular}

Displacement of the central point of the loaded edge in the direction of the in-plane and out-of-plane loads.
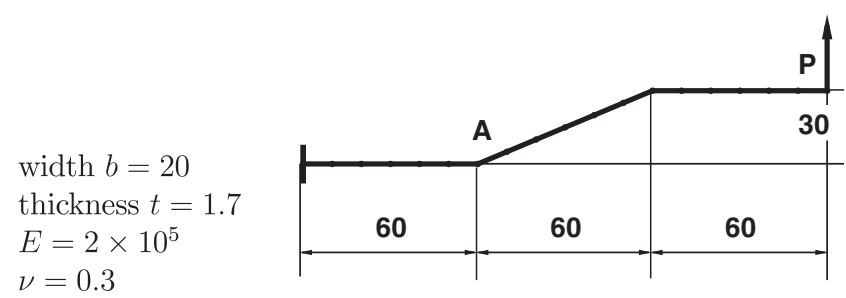

Figure 6. Z-shaped cantilever. Geometry.
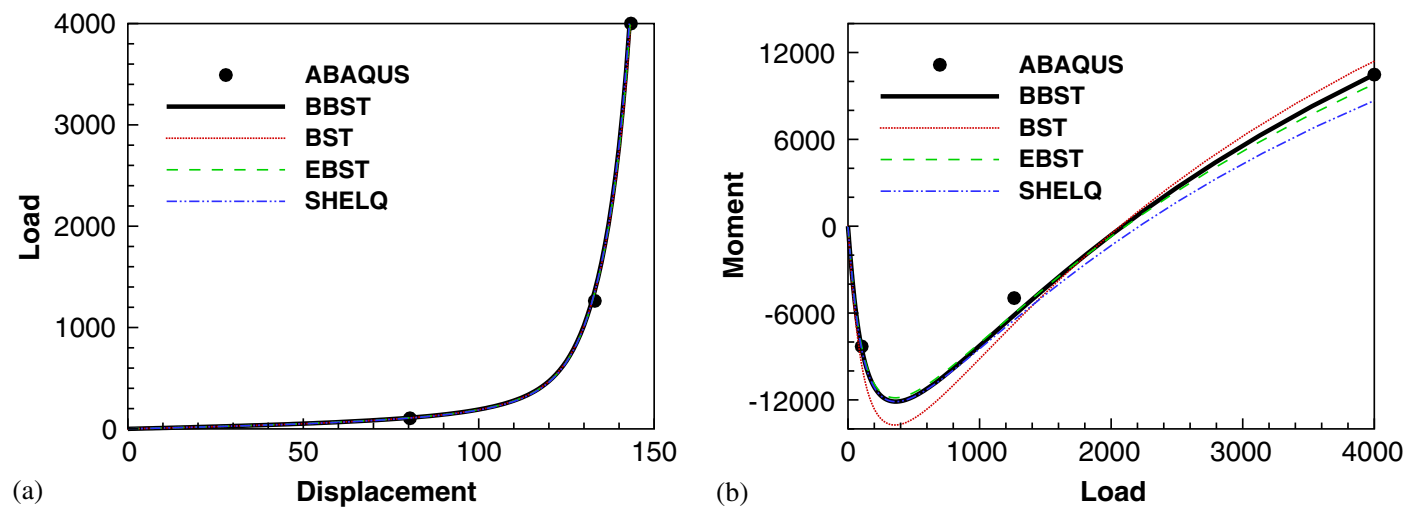

Figure 7. Z-shaped cantilever: (a) load vs displacement; and (b) bending moment at A vs load.

\subsection{Z-shaped cantilever}

This example was extracted from a set of benchmarks for geometric non-linear behaviour of structures [24]. This benchmark is intended to assess membrane and bending actions, tension stiffening and change in sign of bending moments under large rotations and large displacements. Figure 6 shows the cross section properties, the material parameters and the initial geometry of a Z-shaped cantilever under a conservative load at the free end.

In the discretization 12 divisions along each part have been chosen. In Figure 7(a) the vertical displacement of the point where the load is applied is plotted against the load factor while Figure 7(b) shows the bending moment at point A (see Figure 6) in terms of the load. The 
results obtained are compared with target results obtained with the commercial finite element code ABAQUS [25] and with a quadratic transverse shear deformable shell element (SHELQ) [26]. Also, for reference, the results obtained with the BST [13] and EBST elements [15] are included. The bending moment at point $\mathrm{A}$ is computed as the average of the bending moments computed at integration points in the neighbourhood of $\mathrm{A}$. The results show an excellent agreement with the expected results for the mesh used.

\subsection{Z-section cantilever}

This is a benchmark also recommended by NAFEMS [27]. A Z-section cantilever under a 1.2 $\mathrm{MN} \mathrm{m}$ torque at its free end. The torque is applied by two shear forces of $0.6 \mathrm{MN}$ uniformly distributed over each flange (Figure 8). A linear elastic material with Young's modulus $E=210 \mathrm{GPa}$ and Poisson's ratio $v=0.3$ is considered. The shell thickness is $t=0.1 \mathrm{~m}$. The target solution is the axial stress $\sigma_{x x}=-108 \mathrm{MPa}$ at the mid-surface of point A.

Two meshes were considered. A relatively coarse mesh of 96 elements (eight divisions in the length and two at each plane part across the section) and a fine mesh with 960 elements (32 divisions in the length and 5 at each part across the section). The results obtained are $\sigma_{x x}=-95.3 \mathrm{MPa}(-11.8 \%)$ and $\sigma_{x x}=-106.3 \mathrm{MPa}(-1.6 \%)$ for the coarse and the fine meshes, respectively. These values are obtained by extrapolations from the four nearest Gauss points. The same problem appears in ABAQUS user's manual [25] solved using the fine mesh with the three variants of a quadrilateral element (S4R, S4RS and S4RSW). They report a value of $\sigma_{x x}=-100.3 \mathrm{MPa}(-7.1 \%)$ and a slow convergence as the mesh is refined. We note that the analysis with ABAQUS required twice the number of DOFs used with the BBST element. Note also that the $90^{\circ}$ folds in the geometry preclude a reliable use of the BST and EBST elements developed under the assumption of smooth surfaces.

\subsection{Branching shell of revolution}

A branching shell is considered to assess the element behaviour when more than two elements share a side. Figure 9(a) shows the geometry of the shell. The thickness is different for the three shells that compose the structure. The material is isotropic with $E=10^{7}$ and $v=0.3$. The spherical dome and the lower cylinder are subjected to internal pressure $P=1000$, that is equilibrated by equal forces applied at the ends of the cylinder.

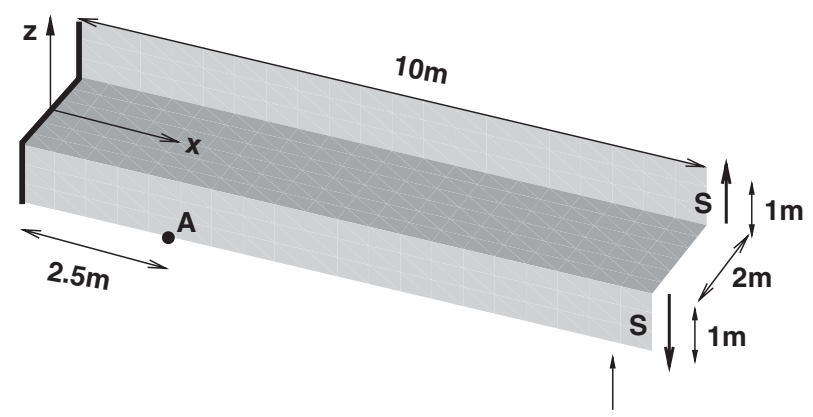

Figure 8. Z-section cantilever. Geometry. 


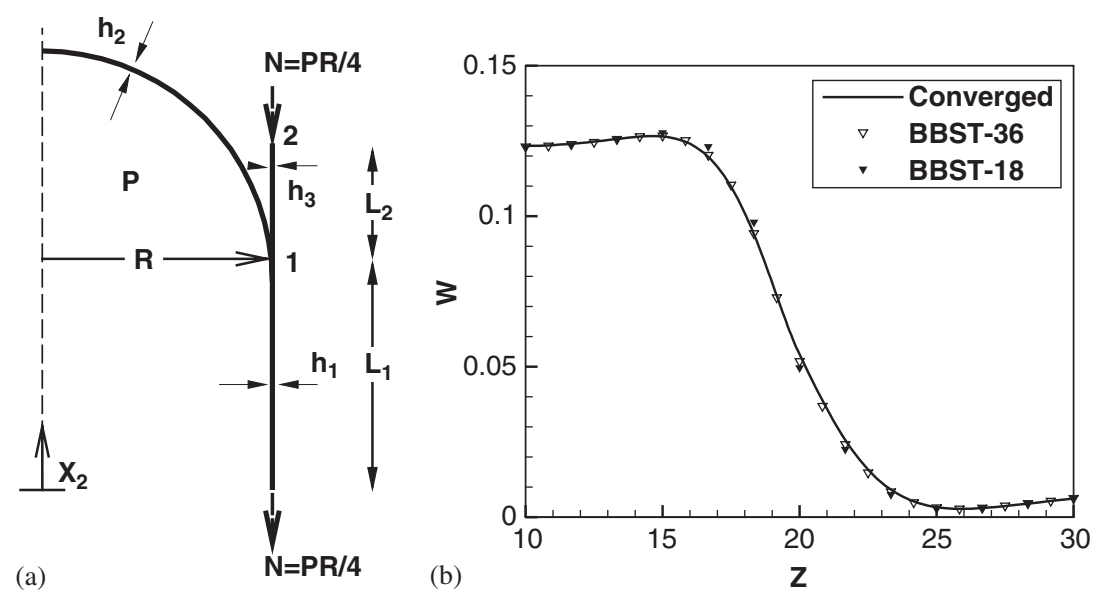

Figure 9. Krauss's ramified shell: (a) geometry $R=20, L_{1}=20, L_{2}=10, h_{1}=0.3, h_{2}=0.4$, and $h_{3}=0.5$; and (b) normal displacement along the cylinder.

Only one quarter of the geometry was discretized due to symmetry using two different meshes. In both cases the spherical dome was modelled with 648 elements (36 elements along the joint, i.e. one element every $2.5^{\circ}$ ). The finest mesh included 864 elements for the upper cylinder and 1728 for the lower cylinder, uniformly spaced along the meridian (12 elements in the upper cylinder and 24 in the lower part). The coarser mesh has exactly half the elements and is also uniformly spaced along the meridian (6 elements in the upper part and 12 in the lower part). Figure 9(b) shows the displacement normal to the cylinder wall. Present results are compared with a converged finite element solution [28] (an analytical solution is also possible). It can be seen that even for the coarser mesh (18 elements along the cylinder, BBST-18) the numerical results are in very good agreement with the converged solution.

\subsection{Straight cellular bridge}

This example studies a box-girder straight bridge analysed in Reference [29]. Figure 10 shows the bridge cross section and the material properties. The bridge spans a length of $40 \mathrm{~m}$ and the ends have the cross section in-plane displacements restrained while the axial displacements are free.

A concentrated force $P=1000 \mathrm{kN}$ is alternatively applied at points $\mathrm{C}$ (centre) and B (box edge). Due to symmetry only half the span was modelled using 20 elements in that direction and 30 elements in the cross section leading to a total of 1200 elements and 630 nodes. The same problem was analysed with the code ABAQUS with the same node positions but using the shell element S4 (four-node quadrilateral). For comparative purposes the bridge was also modelled using eight-noded solid elements. In this case the cross section was discretized using 64 elements (two elements across the thickness) and the total mesh includes 1280 elements and 4109 nodes.

The vertical displacements at the central cross section (upper and lower surfaces) have been plotted in Figure 11. As expected the modellization using brick elements is stiffer than using shell elements, but it can be seen that the displacement pattern is identical. The comparison of the present element with the ABAQUS element S4 shows also identical displacement patterns but the present element is slightly more flexible. 


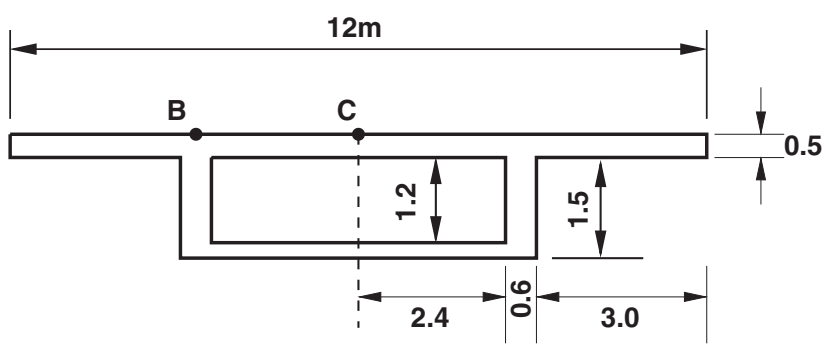

Figure 10. Box-girder bridge under a concentrated load. Cross section geometry. $E=25 \mathrm{GPa}, v=15, L=40 \mathrm{~m}$.
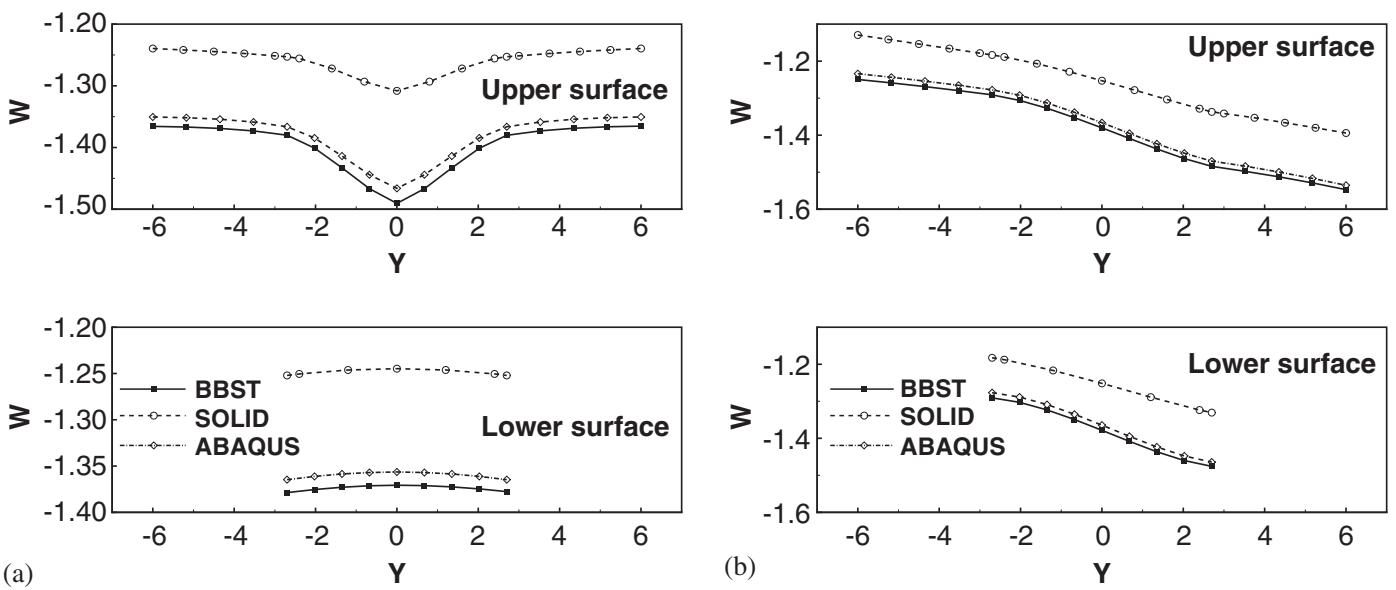

Figure 11. Box girder straight bridge under a point load. Vertical displacements at the central cross section: (a) load at point $\mathrm{C}$; and (b) load at point B.

\subsection{Curved bridge with cellular cross section}

This example corresponds to the analysis of a curved bridge with a cellular cross section simple supported at the ends (cross section in-plane displacements are constrained). This structure has been previously analysed in References $[29,30]$ using the finite strip method in both cases. Figure 12(a) shows the details of the cross section and the material properties. The bridges spans an angle of one radian, the radius at the centre of the bridge is $30.1 \mathrm{~m}$.

A point load of $P=1000 \mathrm{kN}$ is applied at the centre. Half the bridge span has been discretized with 10 divisions in that direction, whilst 34 elements were included in the cross section, leading to 363 nodes and 680 elements. Results with the present formulation are compared with those obtained with the program ABAQUS using the quadrilateral element S4 and the same distribution of nodes. Figure 12(b) plots the deformed configurations at the bridge centre. Almost identical results are obtained.

\subsection{Buckling of a column with self-contact}

This last example illustrates the buckling of a column compressed between two rigid platens. The column has an X-shaped section. The top platen is pushed and rotated in $7 \mathrm{~ms}$ to buckle the column. 


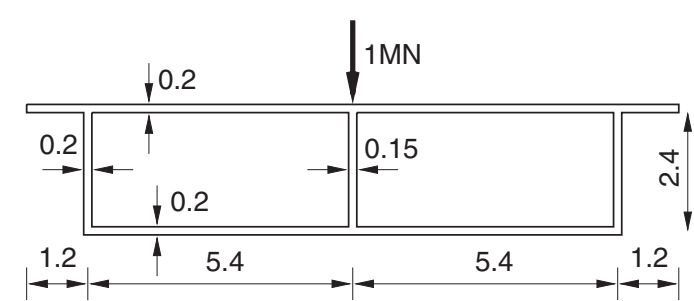

(a)

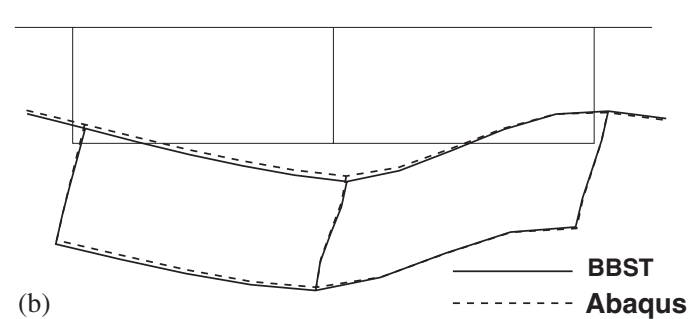

Figure 12. Curved bridge under point load: (a) cross section geometry. $E=25 \mathrm{GPa}, v=0.15, R=30.1 \mathrm{~m}$, angle $=1 \mathrm{rad}$; and (b) deformed configuration at the centre.

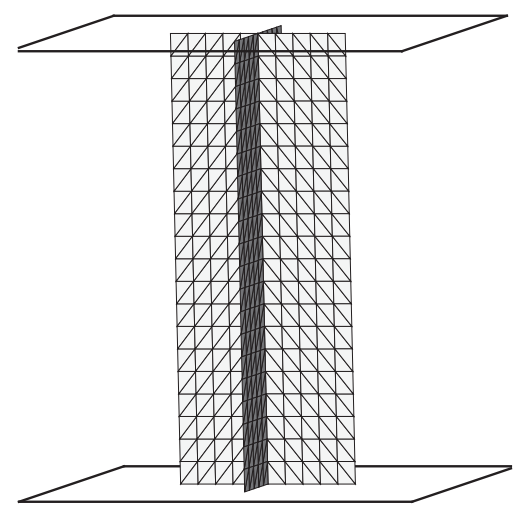

(a)
Initial configuration

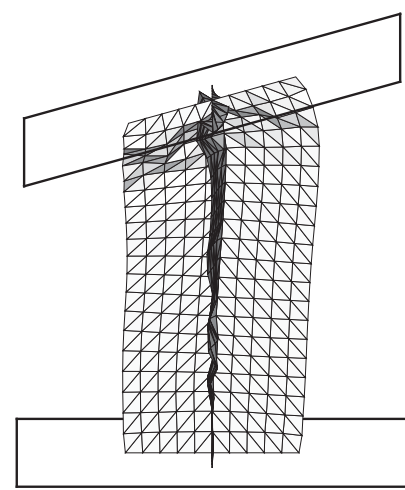

(b) Deformed shape at $\mathbf{3 . 5} \mathrm{msec}$

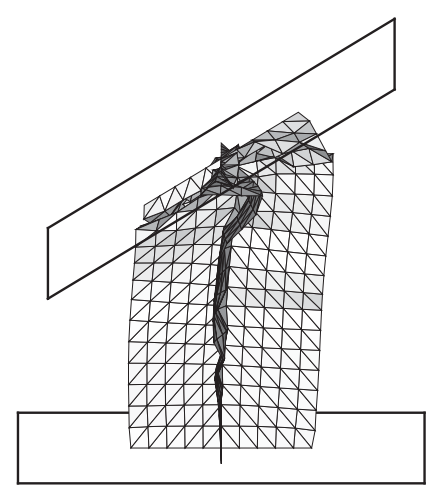

(c) Deformed shape at $7.0 \mathrm{msec}$

Figure 13. Buckling of X-shaped column: (a) initial configuration; (b) deformed shape at $3.5 \mathrm{~ms}$; and (c) deformed shape at $7.0 \mathrm{~ms}$.

The material is steel, with a Young's modulus of $200 \mathrm{GPa}$, a Poisson's ratio 0.3 and a density of $7850 \mathrm{~kg} / \mathrm{m}^{3} . J_{2}$ plasticity is considered with initial yield $\sigma_{o}=250 \mathrm{MPa}$ and linear isotropic hardening $\sigma_{y}^{\prime}=450 \mathrm{MPa}$. The top platen moves vertically with a constant velocity of $50 \mathrm{~m} / \mathrm{s}$ and rotates around the $y$ axis with a velocity of $78.54 \mathrm{rad} / \mathrm{s}$ (final angle $31.5^{\circ}$ ). Figure 13 (a) shows the initial configuration. The mesh has 441 nodes and 800 elements. Figures 13(b) and (c) show the deformed configurations at the middle and at the end of the process.

This problems was taken from the ABAQUS example manual. One of the main aspects to be considered is contact with the platens and self-contact of the shell. This problem was analysed with the program STAMPACK [23] using an explicit dynamic scheme to integrate the momentum equations. The deformed configurations shown and those obtained with the element S4R of ABAQUS are very similar.

\section{CONCLUSIONS}

A triangular finite element formulation for non-linear, large strain analysis of tridimensional shells has been presented. Smooth, kinked and branching situations have been considered. The main 
characteristic of the triangular element formulation is that it does not include rotational degrees of freedom (rotation-free triangular shell element). The element curvature is computed resorting to the nodal positions of the neighbour elements. The numerical examples show that the element converges to the correct solution in all cases, and that the formulation can handle non-smooth surfaces and branching situations. The new rotation-free shell triangle has given excellent results for linear and non-linear problems, including plasticity with large strains, contact with friction, different boundary conditions and loads.

\section{APPENDIX A}

The following development justifies expression (22):

$$
\begin{gathered}
\boldsymbol{\varphi}_{{ }_{n}}^{(i)}=\left(\boldsymbol{\varphi}_{\prime}^{(i)} \cdot \mathbf{n}^{(i)}\right) \mathbf{n}^{(i)}+\left(\boldsymbol{\varphi}_{\prime n}^{(i)} \cdot \mathbf{s}^{(i)}\right) \mathbf{s}^{(i)} \\
\boldsymbol{\varphi}_{{ }_{n}}^{(i)} \cdot \mathbf{t}^{(M)}=\left(\boldsymbol{\varphi}_{\prime}^{(i)} \cdot \mathbf{n}^{(i)}\right) \mathbf{n}^{(i)} \cdot \mathbf{t}^{(M)}+\left(\boldsymbol{\varphi}_{n}^{(i)} \cdot \mathbf{s}^{(i)}\right) \mathbf{s}^{(i)} \cdot \mathbf{t}^{(M)}
\end{gathered}
$$

where $\mathbf{n}^{(i)}$ is the outward normal to the side in the plane of the adjacent element and $\mathbf{s}^{(i)}$ is the side direction. As $\mathbf{s}^{(i)}$ is orthogonal to $\mathbf{t}^{(M)}$ and because they are unit vectors $\left\|\mathbf{t}^{(M)}\right\|=\left\|\mathbf{n}^{(i)}\right\|=1$,

$$
\begin{aligned}
\boldsymbol{\varphi}_{{ }_{n}}^{(i)} \cdot \mathbf{t}^{(M)} & =\left(\boldsymbol{\varphi}_{\prime n}^{(i)} \cdot \mathbf{n}^{(i)}\right) \mathbf{n}^{(i)} \cdot \mathbf{t}^{(M)} \\
& =\left(\boldsymbol{\varphi}_{\prime_{n}}^{(i)} \cdot \mathbf{n}^{(i)}\right) \sin \left(2 \gamma_{i}\right)
\end{aligned}
$$

Besides that

$$
\begin{gathered}
\mathbf{t}^{(i)}=\lambda^{(i)}\left[\boldsymbol{\varphi}_{{ }_{n}}^{(i)} \times \boldsymbol{\varphi}_{{ }_{s}}^{(i)}\right]=\lambda^{(i)} \lambda_{s_{i}}\left[\boldsymbol{\varphi}_{{ }_{n}}^{(i)} \times \mathbf{s}^{(i)}\right] \\
\mathbf{t}^{(i)} \cdot \mathbf{t}^{(i)}=1=\frac{1}{\lambda_{n}^{(i)}}\left[\boldsymbol{\varphi}_{{ }_{n}}^{(i)} \times \mathbf{s}_{i}\right] \cdot \mathbf{t}^{(i)}=\frac{1}{\lambda_{n}^{(i)}} \boldsymbol{\varphi}_{{ }_{n}}^{(i)} \cdot\left[\mathbf{s}_{i} \times \mathbf{t}^{(i)}\right]
\end{gathered}
$$

being $\mathbf{s}_{i}$ and $\mathbf{t}^{(i)}$ orthogonals and $\mathbf{n}^{(i)}=\mathbf{s}^{(i)} \times \mathbf{t}^{(i)}$, it finally yields for moderate membrane distorsions

$$
\begin{aligned}
\boldsymbol{\varphi}_{n}^{(i)} \cdot \mathbf{n}^{(i)} & \simeq \lambda_{n}^{(i)} \\
\boldsymbol{\varphi}_{n}^{(i)} \cdot \mathbf{t}^{(M)} & =\lambda_{n}^{(i)} \sin \left(2 \gamma_{i}\right)
\end{aligned}
$$

\section{ACKNOWLEDGEMENTS}

The first author is a member of the scientific staff of the Science Research Council of Argentina (CONICET). The support provided by a grant of CONICET is gratefully acknowledged. Financial support from CIMNE and support from Quantech ATZ (Barcelona-Spain) by providing the code Stampack, are also acknowledged. 


\section{REFERENCES}

1. Ugural AC. Stresses in Plates and Shells. McGraw Hill: New York, 1981.

2. Bushnell D, Almroth BO. Finite difference energy method for non linear shell analysis. Journal of Computers and Structures 1971; 1:361.

3. Bushnell D. Computerized analysis of shells-governing equations. Journal of Computers and Structures 1984; 18(3):471-536.

4. Nay RA, Utku S. An alternative to the finite element method. Variational Methods Engineerings 1972; 1:62-74.

5. Barnes MR. Form finding and analysis of tension space structures by dynamic relaxation. Ph.D. Thesis, Department of Civil Engineering, The City University, London, 1977.

6. Hampshire JK, Topping BHV, Chan HC. Three node triangular elements with one degree of freedom per node. Engineering Computations 1992; 9:49-62.

7. Phaal R, Calladine CR. A simple class of finite elements for plate and shell problems. II: An element for thin shells with only translational degrees of freedom. International Journal for Numerical Methods in Engineering 1992; 5:979-996.

8. Oñate E, Cervera M. Derivation of thin plate bending elements with one degree of freedom per node. Engineering Computations 1993; 10:553-561.

9. Rio G, Tathi B, Laurent H. A new efficient finite element model of shell with only three degrees of freedom per node. Applications to industrial deep drawing test. In Recent Developments in Sheet Metal Forming Technology, Barata Marques MJM (ed.). 18th IDDRG Biennial Congress, Lisbon, 1994.

10. Brunet M, Sabourin F. Prediction of necking and wrinkles with a simplified shell element in sheet forming. In International Conference of Metal Forming Simulation in Industry, Kröplin B (ed.), vol. II, 1994; $27-48$.

11. Oñate E, Zárate F. Rotation-free plate and shell triangles. International Journal for Numerical Methods in Engineering 2000; 47:557-603.

12. Cirak F, Ortiz M. Fully $C^{1}$-conforming subdivision elements for finite deformations thin-shell analysis. International Journal for Numerical Methods in Engineering 2001; 51:813-833.

13. Flores FG, Oñate E. A basic thin shell triangle with only translational DOFs for large strain plasticity. International Journal for Numerical Methods in Engineering 2001; 51:57-83.

14. Oñate E, Cendoya P, Miquel J. Non linear explicit dynamic analysis of shells using the BST rotation-free triangle. Engineering Computations 2002; 9(6):662-706.

15. Flores FG, Oñate E. Improvements in the membrane behaviour of the three node rotation-free BST shell triangle using an assumed strain approach. Computer Methods in Applied Mechanics and Engineering 2005; 194:907-932.

16. Brunet M, Sabourin F. Analysis of a rotation-free 4-node shell element. International Journal for Numerical Methods in Engineering 2006; 66(9):1483-1510.

17. Oñate E, Flores FG. Advances in the formulation of a rotation-free basic shell triangle. Computer Methods in Applied Mechanics and Engineering 2005; 194:2406-2443.

18. Büchter N, Ramm E. Shell theory versus degeneration-a comparison in large rotation finite element analysis. International Journal for Numerical Methods in Engineering 1992; 34:39-59.

19. Hauptmann R, Schweizerhof K. A systematic development of solid-shell element formulations for linear and nonlinear analysis employing only displacement degrees of freedom. International Journal for Numerical Methods in Engineering 1998; 42:49-69.

20. Belytschko T, Stolarski H, Carpenter N. A $C^{0}$ triangular plate element with one point quadrature. International Journal for Numerical Methods in Engineering 1984; 20:787-802.

21. Flores FG, Oñate E. Rotation-free element for the non-linear analysis of beams and axisymmetric shells. Computer Methods in Applied Mechanics and Engineering 2006; in press.

22. Zienkiewicz OC, Taylor RL. The Finite Element Method. Vol. II: Solid Mechanics. Butterworth-Heinemann: Stoneham, MA, 2000.

23. STAMPACK. A General Finite Element System for Sheet Stamping and Forming Problems, ver. 6.0.0. Quantech ATZ: Barcelona, Spain, (www.quantech.es), 2004.

24. Prinja NK, Clegg RA. A review of benchmark problems for geometric non-linear behaviour of 3-D beams and shells. NAFEMS R0024, 1993.

25. Hibbit, Karlson and Sorensen Inc., ABAQUS, ver. 6.4 .2 (student edn). Pawtucket: U.S.A., 2003.

26. Simo JC, Fox DD, Rifai MS. On stress resultant geometrically exact shell model. Part III: Computational aspects of the nonlinear theory. Computer Methods in Applied Mechanics and Engineering 1990; 79:21-70.

27. National Agency for Finite Element Methods and Standards (U.K.). The Standard NAFEMS Benchmarks. Test LE5 of the Publication TNSB, Rev. 3, October 1990. 
28. Flores FG, Godoy LA. Finite element applications to the internal pressure loadings on spherical shells and other shells of revolution. In Finite Element Analysis of Thin Walled Structures (Chapter 9), John Bull (ed.). Barking, England, 1990; 259-296.

29. Oñate E, Suárez B. A unified approach for the analysis of bridges, plates and axisymmetric shells using the linear Mindlin strip element. Computers and Structures 1986; 17(3):407-426.

30. Cheung YK. Finite Strip Method in Structural Analysis. Pergamon: Oxford, 1976. 